DOE/ER/14079-36

CWP-162

February 1995

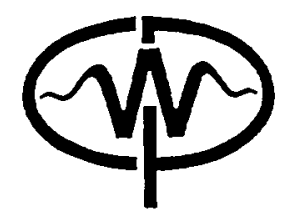

\title{
Transformation to Zero Offset in Transversely Isotropic Media
}

\author{
Tariq Alkhalifah
}

\section{DISCLAIMER}

This report was prepared as an account of work sponsored by an agency of the United States Government. Neither the United States Government nor any agency thereof, nor any of their employees, makes any warranty, express or implied, or assumes any legal liability or responsibility for the accuracy, completeness, or usefulness of any information, apparatus, product, or process disclosed, or represents that its use would not infringe privately owned rights. Reference herein to any specific commercial product, process, or service by trade name, trademark, manufacturer, or otherwise does not necessarily constitute or imply its endorsement, recommendation, or favoring by the United States Government or any agency thereof. The views and opinions of authors expressed herein do not necessarily state or reflect those of the United States Government or any agency thereof.

\section{MASTER}

Center for Wave Phenomena

Colorado School of Mines

Golden, Colorado 80401

303/273-3557

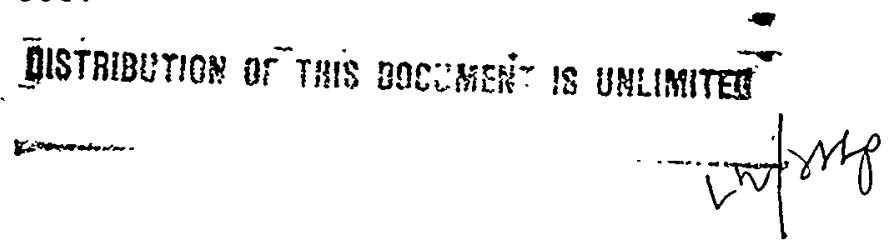




\title{
Transformation to Zero Offset in Transversely Isotropic Media
}

\author{
Tariq Alkhalifah
}

\begin{abstract}
Nearly all dip moveout correction (DMO) implementations to date assume isotropic homogeneous media. Usually, this has been acceptable considering the tremendous cost savings of homogeneous isotropic DMO and considering the difficulty of obtaining the anisotropy parameters required for effective implementation. In the presence of typical anisotropy, however, ignoring the anisotropy can yield inadequate results.

Since anisotropy may introduce large deviations from hyperbolic moveout, accurate transformation to zero-offset in anisotropic media should address such nonhyperbolic moveout behavior of reflections. Artley and Hale's (1994) $v(z)$ ray tracing-based DMO, developed for isotropic media, provides an attractive approach to treating such problems. By using a ray-tracing procedure crafted for anisotropic media, I modify some aspects of Artley and Hale's DMO so that it can work for $v(z)$ anisotropic media.

DMO impulse responses in typical transversely isotropic (TI) models deviate substantially from the familiar elliptical shape associated with responses in homogeneous isotropic media (to the extent that triplications arise even where the medium is homogeneous). Such deviations can exceed those caused by vertical inhomogeneity, thus emphasizing the importance of taking anisotropy into account in DMO processing.

For isotropic or elliptically anisotropic media, the impulse response is an ellipse, but as the key anisotropy parameter $\eta$ (Alkhalifah and Tsvankin, 1995) varies, the shape of the response differs substantially from elliptical. For typical $\eta>0$, the impulse response in TI media tends to broaden compared to the response in an isotropic homogeneous medium, a behavior opposite to that encountered in typical $v(z)$ isotropic media, where the response tends to be squeezed. Furthermore, the amplitude distribution along the DMO operator differs significantly from that for isotropic media.

Application of this anisotropic DMO to data from offshore Africa resulted in a considerably better alignment of reflections from horizontal and dipping reflectors in common-midpoint gather than that obtained using an isotropic DMO. Even the presence of vertical inhomogeneity in this medium could not eliminate the importance of considering the shale induced anisotropy.
\end{abstract}




\section{DISCLAIMER}

\section{Portions of this document may be illegible in electronic image products. Images are produced from the best available original document.}




\section{INTRODUCTION}

The process of transforming prestack data to zero offset (TZO) accomplishes moveout correction for reflections from horizontal and dipping reflectors. Specifically, in an isotropic, homogeneous medium, TZO includes both normal moveout correction (NMO) and dip moveout correction (DMO). I use the term TZO to describe the one-step ray-tracing process used here to map nonzero-offset traveltimes directly to zero-offset times. Therefore, DMO correction corresponds to TZO with the moveout correction for horizontal reflectors removed.

For homogeneous isotropic media, reflections from dipping, as well as horizontal, reflectors have hyperbolic moveout. In $v(z)$ isotropic media, the moveout has additional nonhyperbolic terms. These additional terms are generally small and usually ignored in the conventional NMO and DMO process. On the other hand, the nonhyperbolic moveout terms for typical homogeneous transversely isotropic media (TI) with vertical symmetry axis (Alkhalifah, 1995) are comparably larger than those in isotropic $v(z)$ media, and should be corrected for in the NMO and DMO steps.

Extensions of DMO algorithms to anisotropic media have been discussed in several papers. Uren et al. (1990) showed that the DMO method of Forel-Gardner (1988) can be modified to handle, in a straightforward way, elliptically anisotropic models. However, elliptical anisotropy is a special case of transverse isotropy that is not typical of the subsurface (Thomsen, 1986). Anderson and Tsvankin (1995) extended the dipmoveout method developed by Hale (1984) to treat TI media using the analytical formulation for NMO velocity as a function of reflector dip given by Tsvankin (1995). Nevertheless, Hale's original DMO, as well as the anisotropic version, is slow, and the process is based on hyperbolic moveout, which, as we shall see, is invalid for typical TI media. Although Anderson and Tsvankin (1995) show that nonhyperbolic moveout is small for dipping reflectors, it is significant for horizontal and mildly dipping reflectors.

The combination of anisotropic ray tracing ( $\dot{C}$ ervený, 1972) and dip-decomposition (Jakubowicz, 1990) in transforming prestack data to zero-offset (or in applying DMO) is particularly well suited to handling the nonhyperbolic moveout in homogeneous or inhomogeneous TI media. For such media, with accurate traveltime computations, such as by ray tracing, data can be transformed to zero-offset, thus lessening the need to mute the far offsets. Artley and Hale (1994) have shown that such a DMO method is efficient for isotropic media, and I find that it is efficient for anisotropic media as well.

To give an idea of the importance of considering anisotropy in seismic processing, let us revisit a demonstration by Alkhalifah and Tsvankin (1995). Figure 1 shows constant-velocity stacks after applying normal moveout correction, with each of several constant stacking velocities, followed by isotropic homogeneous DMO (such DMO is independent of velocity). A goal of DMO processing is to focus both horizontal and dipping events on the same velocity panel. However, while reflectors with relatively 
small dip in Figure 1 are imaged best with a stacking velocity of $2200 \mathrm{~m} / \mathrm{s}$, the steep reflector (fault) comes into focus at a much higher velocity $(2400-2450 \mathrm{~m} / \mathrm{s})$. Thus, the conventional DMO processing fails to remove the dip-dependence of stacking velocity. As a result, stacking would produce a weak, poorly-focussed image of the dipping fault plane.

Conventional DMO fails here because the stacking (moveout) velocity increases with dip much faster than in the isotropic media assumed in the DMO algorithm. If the shortcoming of the DMO processing had been caused by a vertical velocity gradient, then the steep event in Figure 1 would have been imaged at a lower stacking velocity than that of the events with small dips (Hale and Artley, 1993). Given that velocity increases with depth, the implication is that, here, anisotropy introduces an error in conventional (isotropic, homogeneous) DMO that is contrary to and stronger than that due to vertical velocity variation.

In homogeneous transversely isotropic media with vertical symmetry axis (VTI media), $P$ - and $S V$-waves (I omit the qualifiers in "quasi- $P$-wave" and "quasi- $S V$ wave" for brevity) can be described by the vertical velocities $V_{P 0}$ and $V_{S 0}$ of $P$ - and $S$-waves, respectively, and two dimensionless parameters $\epsilon$ and $\delta$ (Thomsen, 1986).

$$
\begin{gathered}
\epsilon \equiv \frac{c_{11}-c_{33}}{2 c_{33}}, \\
\delta \equiv \frac{\left(c_{13}+c_{44}\right)^{2}-\left(c_{33}-c_{44}\right)^{2}}{2 c_{33}\left(c_{33}-c_{44}\right)} .
\end{gathered}
$$

Tsvankin and Thomsen (1994) and Alkhalifah and Larner (1994) demonstrated that $P$-wave velocity and traveltime are practically independent of $V_{S 0}$, even for strong anisotropy. This implies that, for practical purposes, $P$-wave kinematic signatures can be considered a function of just three parameters: $V_{P 0}, \delta$, and $\epsilon$.

Alkhalifah and Tsvankin (1995) further demonstrated that a new representation in terms of just two parameters is sufficient for performing all time-related processing, such as normal moveout correction (including non-hyperbolic moveout correction, if necessary), dip-moveout removal, and prestack and poststack time migration. These two parameters are the normal-moveout velocity for a horizontal reflector

$$
V_{\mathrm{nmo}}(0)=V_{p 0} \sqrt{1+2 \delta},
$$

and the anisotropy coefficient $\eta$,

$$
\eta \equiv 0.5\left(\frac{V_{h}^{2}}{V_{\mathrm{nmo}}^{2}(0)}-1\right)=\frac{\epsilon-\delta}{1+2 \delta},
$$

where $V_{h}$ is the horizontal velocity.

Moreover, Alkhalifah and Tsvankin (1995) further show that these two parameters, $\eta$ and $V_{\text {nmo }}(0)$, are obtainable solely from surface seismic $P$-wave data: specifically, from estimates of stacking velocity for reflections from interfaces having two 


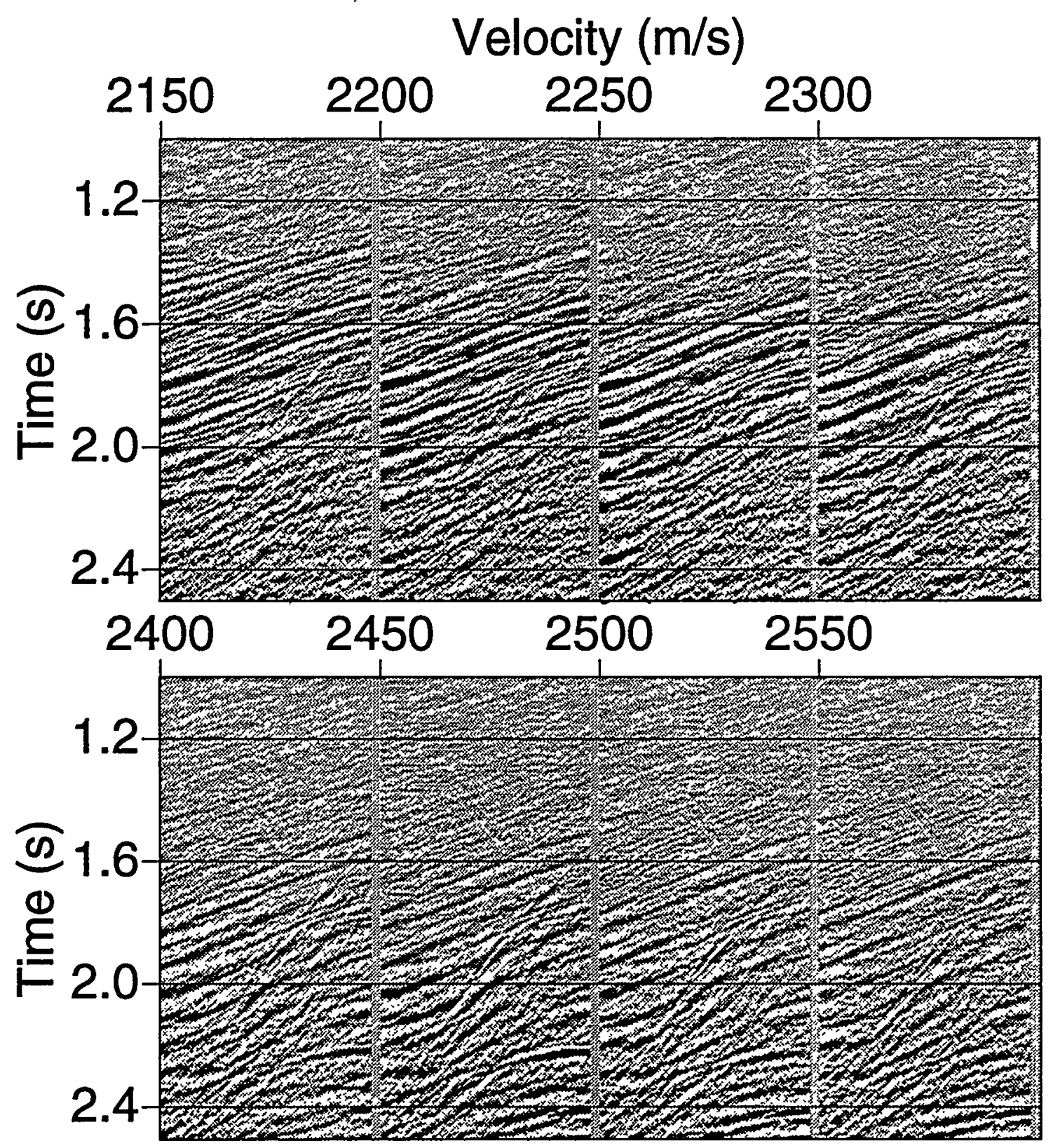

Fig. 1. Constant-velocity stacks for the area below the gray bar in Figure 12 after the conventional sequence of NMO and constant-velocity, isotropic DMO. The velocity values at the top correspond to stacking velocities for horizontal reflectors. 
distinct dips. The third parameter, $V_{P 0}$, is needed for time-to-depth conversion only. The two-parameter representation and inversion also holds in $v(z)$ media. For that situation, these two parameters are expressed in terms of the vertical time $\tau$.

After outlining the TZO method, I generate and study DMO impulse responses in VTI media, and compare them with the familiar elliptical DMO response for isotropic, homogeneous media. Finally, I apply this DMO approach to synthetic and field data, demonstrating the need to take anisotropy into account in order to achieve the goals of DMO.

\section{TRANSFORMATION TO ZERO OFFSET}

The DMO approach of Artley and Hale (1994), appropriate for isotropic $v(z)$ media, is based on a combination of Jakubowicz's (1990) dip-decomposition method and ray tracing in isotropic $v(z)$ media. Their DMO algorithm results from subtracting an NMO correction after transformation to zero offset. The advantage of using DMO, as opposed to full TZO, is that the separately-applied NMO correction can accommodate smooth lateral velocity variations in the media, while the full TZO treats only $v(z)$ media.

The method of Artley and Hale requires solution of a system of nonlinear equations to find a trio of rays needed to calculate the zero-offset time. This ray trio includes the rays from source, receiver, and zero-offset locations to each (potential) specular reflection point in the subsurface. Solution of a system of five equations and five unknowns is necessary in order to obtain the ray trio. In particular, one of these equations is based on Snell's law, which requires that the incident and reflection angles be equal. That is,

$$
\theta_{0}=\frac{\theta_{s}+\theta_{g}}{2},
$$

where $\theta_{0}$ is the angle between the normal to the reflector and the vertical axis (the reflector dip), $\theta_{s}$ is the angle between the incident ray originating from the source and the vertical, and $\theta_{g}$ is the angle between the reflected ray and the vertical. A detailed description of the algorithm is given by Artley and Hale (1994).

Using ray tracing for anisotropic media, as described in Appendix $A$, and replacing equation (3) with

$$
\frac{\sin \left(\theta_{s}-\theta_{0}\right)}{V\left(\theta_{s}\right)}=\frac{\sin \left(\theta_{0}-\theta_{g}\right)}{V\left(\theta_{g}\right)}
$$

where $V(\theta)$ is the phase velocity, modifies the TZO of Artley and Hale to accommodate $v(z)$ anisotropic media. Here $\theta$ corresponds to the phase angle, which generally differs from the ray angle in TI media. Because traveltimes and ray parameters are applicable to all midpoints for a $v(z)$ medium, they need to be tabulated only once. As a result, the cost of the modification of the ray-tracing algorithm to treat TI media is negligible compared with the overall cost of the DMO, especially for large data volumes. 
To apply the algorithm as a DMO operation, it is sufficient to subtract from the full TZO traveltime calculations the traveltime portion that corresponds to either NMO correction (Artley and Hale, 1994) or nonhyperbolic moveout correction (Tsvankin and Thomsen, 1994; Alkhalifah and Tsvankin, 1995) for horizontal reflectors. The approach of removing a nonhyperbolic moveout correction is used below to generate DMO impulse responses in VTI media.

Because this method is based on ray tracing, the travel time calculations are exact (within the framework of ray theory), and therefore can appropriately map nonhyperbolic moveout. This is an advantage over DMO methods based on hyperbolic moveout equations (i.e., Anderson and Tsvankin, 1995), which often ignore the large nonhyperbolic moveout in VTI media. This advantage will be exemplified in the discussion of the field data example.

\section{DMO IMPULSE RESPONSES IN VTI MEDIA}

Following Artley and Hale (1994), I use ray tracing (but now in VTI media) to generate exact trajectories for DMO impulse responses in VTI $v(z)$ media. Using this DMO impulse response generator, I study the dependence of the response shape on the various parameters that control DMO in VTI media. These parameters are: $\eta$, $V_{\text {nmo }}(0)$, offset, and NMO-corrected time, $t_{n}$. Let us begin by examining the dependence of the DMO impulse response on the anisotropy, specifically on the parameter $\eta$ in homogeneous VTI media.

\section{Dependence on $\eta$}

The impulse responses in Figure 2 pertain to $V_{\text {nmo }}(0)=2.0 \mathrm{~km} / \mathrm{s}$, offset $=1.5 \mathrm{~km}$, and NMO time, $t_{n}=1.5 \mathrm{~s}$. Superimposed on these black curves is the corresponding DMO trajectory for a homogeneous isotropic or elliptically isotropic medium (gray curves), both of which are characterized by $\eta=0$. Typical $\eta$ values in the subsurface range from -0.1 to 0.3 (Thomsen, 1986; Alkhalifah and Tsvankin, 1995). Departures from the familiar elliptical shape for DMO responses in isotropic media $(\eta=0)$ in this range are evident. It is also interesting to observe the impulse-response behavior for models that are possible, but not typical. For instance, at $\eta=-0.3$, the DMO impulse response looks somewhat like an inverse DMO operator. At the opposite extreme $(\eta=0.6)$, the impulse response is highly flattened, with sizeable triplications at the sides (note again that all models in Figure 2 are homogeneous). However, unlike the triplications encountered in isotropic $v(z)$ media (Artley and Hale, 1994), the triplications here always correspond to reflector dips less than 90 degrees.

The most striking behavior of the impulse responses are the triplications that develop as $\eta$ increases. For positive $\eta$ (i.e., $\eta=0.3$ ), the impulse response in VTI media has three branches. The central one corresponds to the concave-upward region, which includes dips between 0 and $24 \pm 6$ degrees [the upper limit of this range depends on $\eta$ and $\left.V_{\text {nmo }}(0)\right]$. This branch flattens with increase in $\eta$, and can be somewhat 
Distance $(\mathrm{km})$

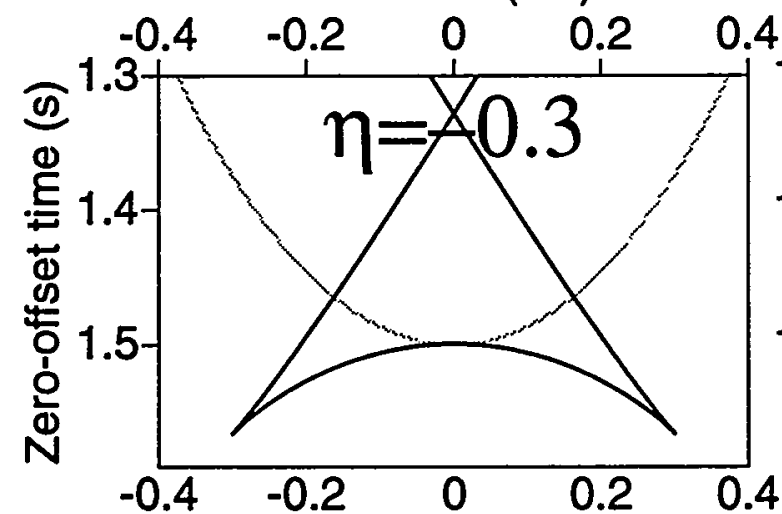

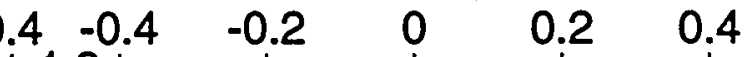
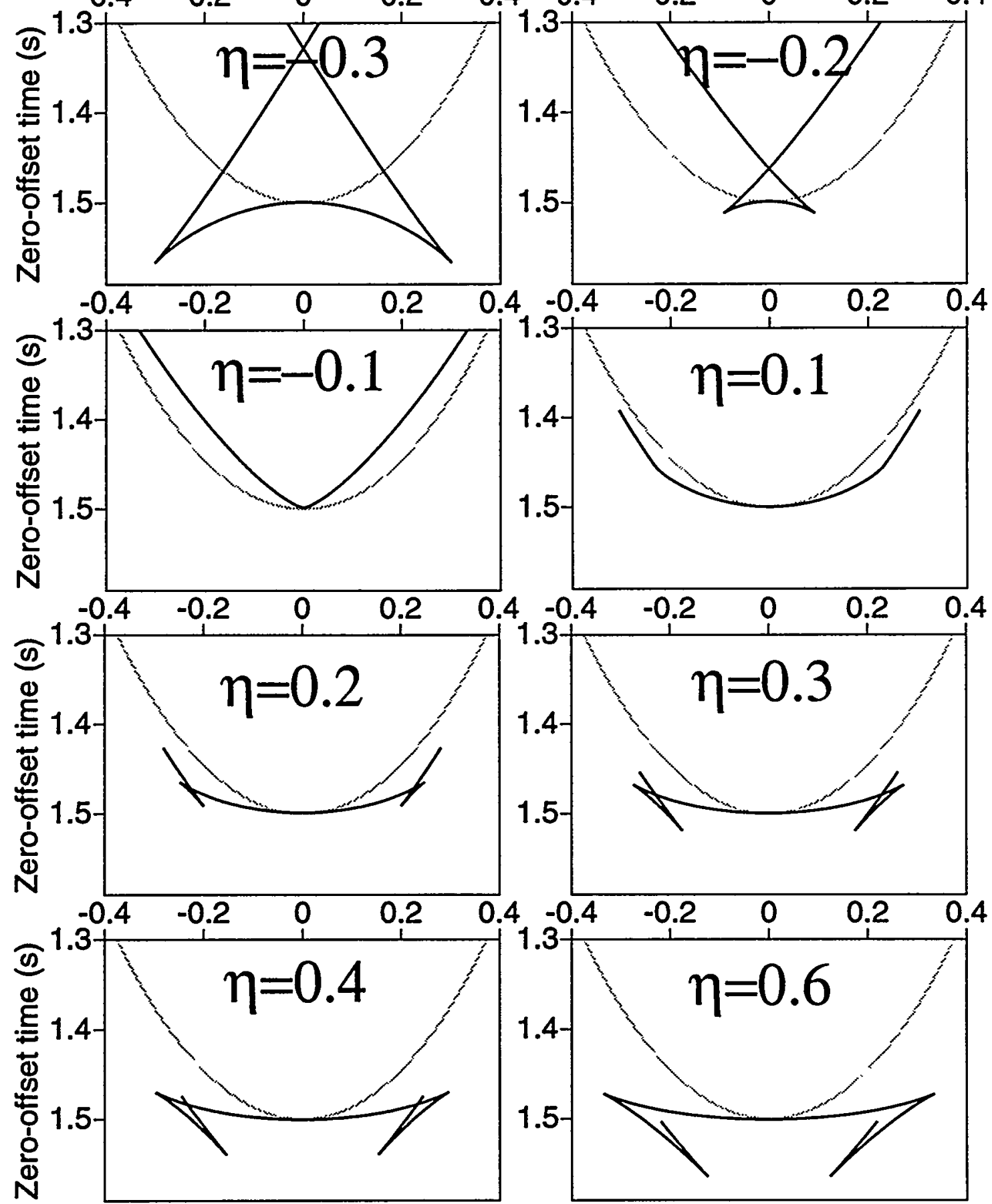

FIG. 2. Shapes of DMO impulse responses in homogeneous VTI media for various $\eta$ values (black curves). Here, offset $=1.5 \mathrm{~km}, t_{n}=1.5 \mathrm{~s}$, and $V_{\text {nmo }}(0)=2.0 \mathrm{~km} / \mathrm{s}$. These curves are superimposed on the corresponding isotropic $(\eta=0)$ constant-velocity DMO trajectories (gray curves). Lateral distance here, and throughout, is measured from the symmetry point of each response. 
approximated by a stretched ellipse. Another branch is seen at the limbs of the previous branch as a concave-downward section of the triplication (this branch reduces to a point for $\eta=0.1$ ). This portion corresponds to dips between $24 \pm 6$ and $48 \pm 5$ degrees; clearly, no flattened ellipse can approximate it. A third branch is represented by a somewhat straight line at the sides of the responses. These lines shift downward with an increase in $\eta$, and this region includes dips between $48 \pm 5$ and 85 degrees. This line would project to the source and receiver locations at the surface, where the associated dip is 90 degrees.

For $\eta<-0.1$, a different kind of triplication develops. This one is centered on the symmetry point, which implies that it acts on reflections from reflectors with small dip. Moreover, negative-sign reflector dips now appear to the right of the impulseresponse symmetry point, instead of the left, as is the case for isotropic media.

As a consequence of the unusual shapes of the DMO operators in VTI media, the energy distributions along the response differ from those in isotropic media. The nonuniform distribution is necessary to insure that the summation of amplitudes along impulse responses in the DMO process yields proper amplitudes of reflections. Figure 3 exemplifies such varying energy distributions and includes a comparison with the isotropic impulse response, given by $\eta=0$. These impulse responses were generated by applying anisotropic DMO, described above, to an input file that contains a single impulse. Clearly, the parts of the response with less curvature have smaller amplitude than do those with more curvature. For example, when $\eta=0.1$, for which the response is flattened (see Figure 2), the reflection from a horizontal reflector in the common-offset domain receives contributions from many adjacent responses, and the amplitude of each contribution must be small enough to insure that the superposition of the contributions for this dip is the same as for all other dips.

Such large differences between the isotropic, homogeneous response and the responses in VTI media imply that the special effort to take anisotropy into account in DMO will be rewarded where data are from VTI media with $\eta \neq 0$.

\section{Dependence on $V_{\text {nmo }}(0)$}

It is well known that the DMO operator for isotropic homogeneous media is independent of velocity. This is not true for VTI media with $\eta \neq 0$. However, as seen in Figure 4, $V_{\text {nmo }}(0)$ has only small influence on the DMO impulse response, and practically no influence on the region of the impulse response corresponding to moderate and mild dips (dips up to 30 degrees). Specifically, the concave-upward branch does not change with a change in $V_{\text {nmo }}(0)$. It only varies in width; that is, with increase in $V_{\text {nmo }}(0)$, the concave-downward and straight-line branches are shifted somewhat inward. This shift arises because for a given reflector dip, slopes on the DMO impulse responses decrease with increase in velocity, and I find that the transition between branches occurs at about the same dip, independent of velocity. In isotropic media, all shifts caused by velocity variation take place along the elliptical operator, and therefore do not alter the shape at all. The range of $V_{\text {nmo }}(0)$ displayed 

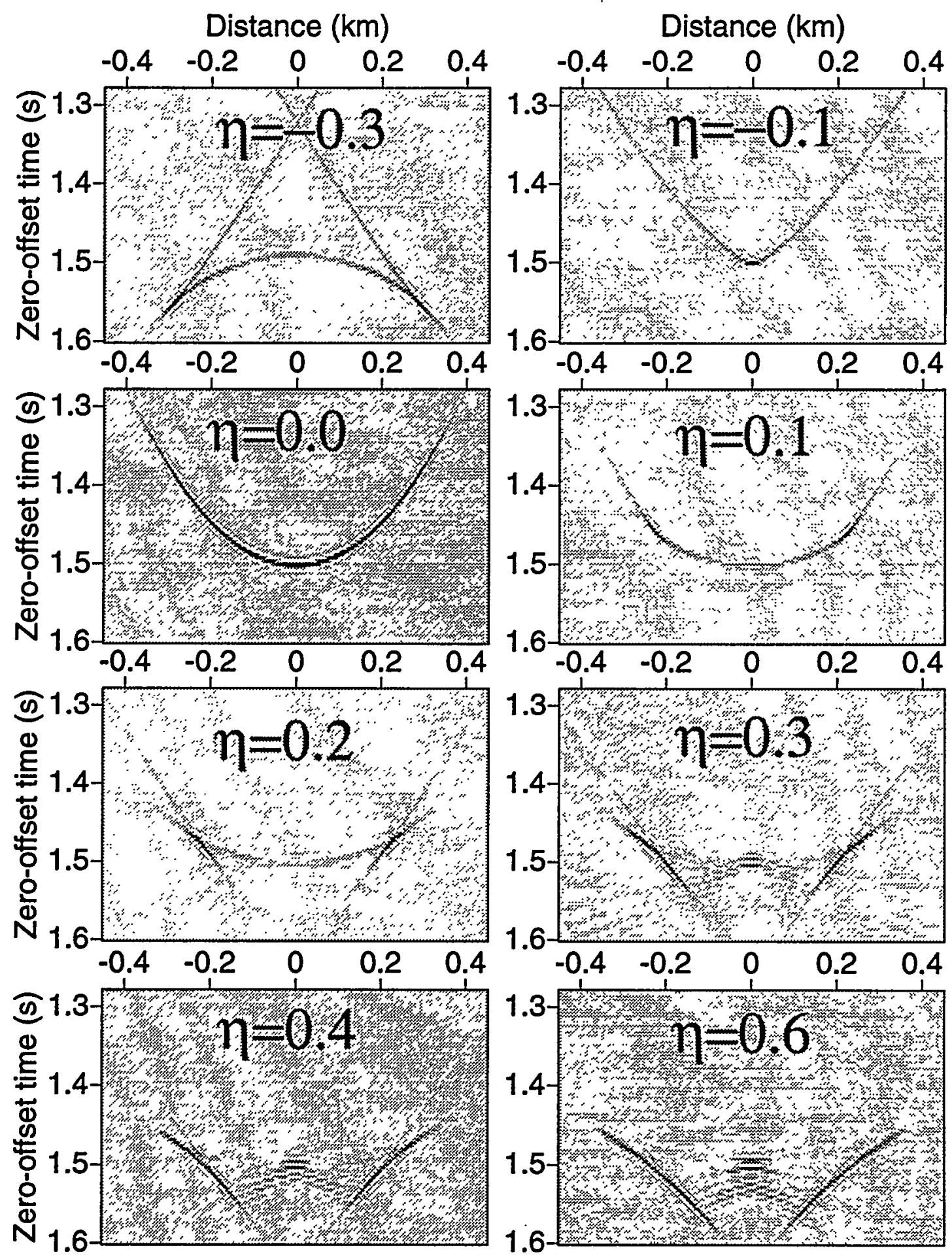

FIG. 3. DMO impulse responses in VTI media for various $\eta$ values. Here offset $=1.5$ $\mathrm{km}, t_{n}=1.5 \mathrm{~s}$, and $V_{\mathrm{nmo}}(0)=2.0 \mathrm{~km} / \mathrm{s}$. 


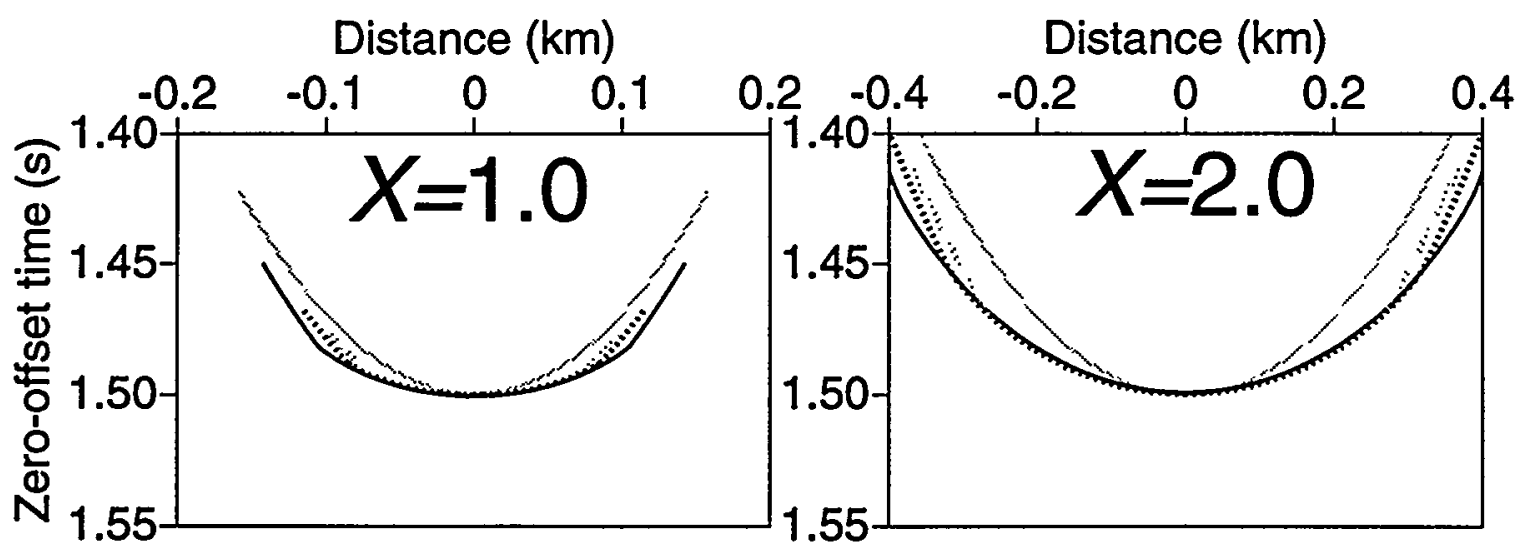

FIG. 4. DMO impulse responses in VTI media with $V_{\text {nmo }}(0)=2.0 \mathrm{~km} / \mathrm{s}$ (solid black curve), $V_{\text {nmo }}(0)=2.5 \mathrm{~km} / \mathrm{s}$ (dashed black curve), and $V_{\text {nmo }}(0)=3.0 \mathrm{~km} / \mathrm{s}$ (dashed gray curve). Here, offset $=1.5 \mathrm{~km}, t_{n}=1.5 \mathrm{~s}$, and $\eta=0.1$. As before, the impulse responses are superimposed on the corresponding isotropic constant-velocity operators (gray curve).

in Figure $4(2.0$ to $3.0 \mathrm{~km} / \mathrm{s})$ is far greater than the range of errors usually associated with measuring $V_{\text {nmo }}(0)$ (i.e., from velocity analysis). This implies that, especially for dips corresponding to the first branch, the DMO process in VTI media is practically independent of the NMO velocity, thus approximating the velocity-independence for isotropic media. This is consistent with the weak-anisotropy approximation of Anderson and Tsvankin (1995).

Thus, of the two key parameters, $V_{\text {nmo }}(0)$ and $\eta$, only $\eta$ has a strong influence on the shape of the DMO impulse response. Therefore, after applying NMO correction to the data, a process of estimating the $\eta$ value that best corrects the moveout to zerooffset can be used. For example, a semblance analysis technique can be developed using successive applications of DMO, each based on a different constant value of $\eta$.

Although $\eta$ forms essentially a one-parameter control on the shape of the impulse response in VTI media, clearly the responses in Figure 2 are not squeezed or stretched versions of the isotropic ones, as in isotropic $v(z)$ media. They exhibit a much more complicated departure from the typical elliptical shape.

\section{Dependence on offset and NMO time}

As is known, the importance of DMO correction increases with offset; i.e., the elliptical impulse responses of the DMO process broaden with increasing offset in homogeneous, isotropic media. Likewise, the impulse responses for VTI media broaden, but, as we have seen, the shapes are not elliptical. The influence of offset on the impulse responses in a homogeneous VTI medium with $\eta=0.1$ is shown in Figure 5. Note that the general shape of the response remains the same with increasing offset; 


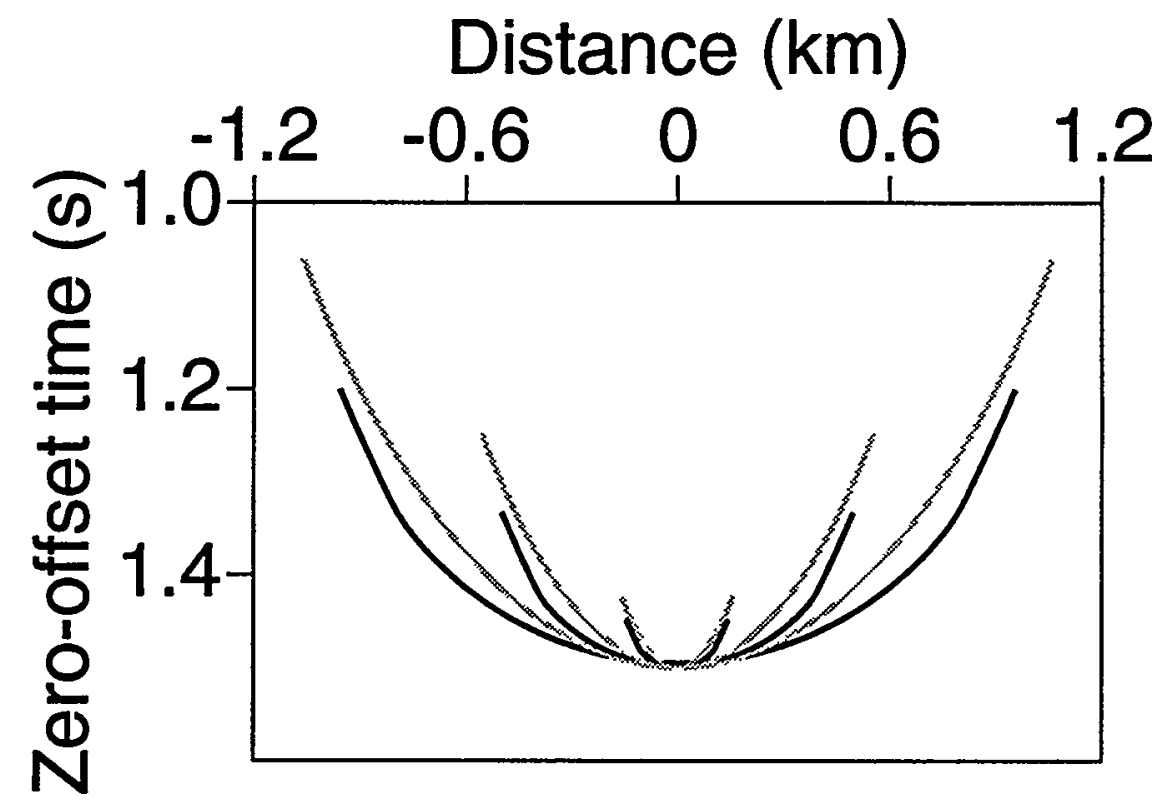

FIG. 5. DMO impulse responses in a VTI medium for three offsets given by $X=1.0$ $\mathrm{km}$ (the narrowest of the responses), $X=2.0 \mathrm{~km}$, and $X=3.0 \mathrm{~km}$ (the widest of the responses). Here, $t_{n}=1.5 \mathrm{~s}, \eta=0.1$, and $V_{\mathrm{nmo}}(0)=2.0 \mathrm{~km} / \mathrm{s}$. The responses of the VTI medium (black) are superimposed on the corresponding isotropic constant-velocity operators (gray). 
however, the departure of the VTI impulse response (black curve) from the isotropic homogeneous operator (gray curve) increases with increasing offset.

The DMO impulse responses in Figure 6, generated for various NMO-corrected times, pertain to an offset of $1.5 \mathrm{~km}$. As with isotropic media, the width of the impulse response decreases with increasing $t_{n}$. However, the departure of the shapes of the impulse responses for VTI media (black curves) from the ones corresponding to homogeneous, isotropic media (gray curves) remains practically the same. In contrast, Artley and Hale (1994) showed that the differences between the impulse responses for isotropic homogeneous and $v(z)$ media decrease with increasing time, to a point where the two responses practically coincide. This implies that, for example, if inhomogeneity was large enough to offset the action of anisotropy in the shallow part of a section, the relative importance of inhomogeneity would reduce with depth.

\section{Dependence on vertical inhomogeneity}

It has been argued that when the subsurface is both anisotropic and vertically inhomogeneous, DMO is best done with an isotropic homogeneous operator (Gonzalez et al., 1992; Larner, 1993; Tsvankin, 1995). The validity of this choice depends on the nature of both anisotropy and inhomogeneity. For example, this approach might be acceptable if anisotropy is weak enough; however, in media with typical values of $\eta$ [i.e., the anisotropy levels usually associated with some shales $(\eta \geq 0.1)$, as we will see later in the field example], the anisotropy influence on the DMO operator exceeds that of the inhomogeneity.

All the examples so far pertain to homogeneous media. Now consider media in which velocities increase with depth. Figure 7 shows impulse responses in VTI media for $\eta=0.1$ and $\eta=0.3$, with the vertical velocity gradient $a$ ranging between 0 and 1.0 $\mathrm{s}^{-1}$. First note that the inhomogeneity introduces an additional branch corresponding to dips mostly beyond 90 degrees. This additional branch is similar to that observed by Artley and Hale (1994) in $v(z)$ isotropic media. For mild to moderate dips (dips up to 30 degrees), differences in responses for inhomogeneous media from those for the homogeneous case $(a=0)$, shown at the top of Figure 7 , are generally small, if not negligible. Other than the additional branch, the shapes of the responses are generally similar to the homogeneous ones.

Figure 8 shows actual impulse responses in $v(z)$ VTI media, with the energy distribution clearly displayed. As with the homogeneous case (Figure 3 ), such irregular energy distribution (e.g., the strong amplitudes where the curvature of the impulse response is largest) depends mainly on the shape of the impulse response.

The similarities between impulse responses in homogeneous and $v(z)$ VTI media, especially for mild and moderate dips, imply that anisotropy has a larger influence on DMO than does inhomogeneity. Therefore, a homogeneous VTI DMO algorithm will likely give acceptable results in typical $v(z)$ media for mild and moderate dips. Where isotropic $v(z)$ DMO impulse responses can be well approximated by squeezed 


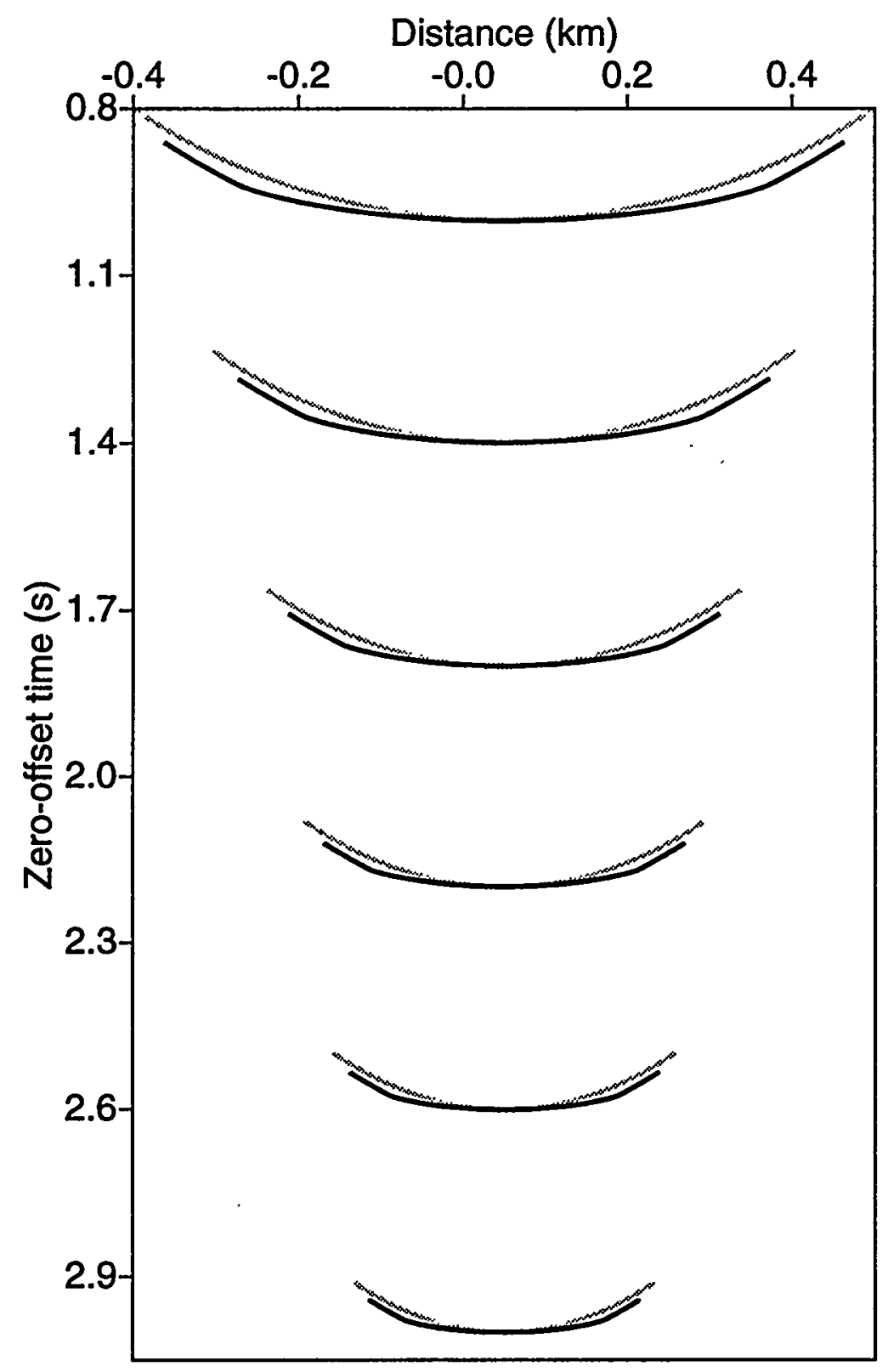

FIG. 6. DMO impulse responses in a VTI medium for scatterers at six different depths (i.e., six different values of $t_{n}$ ). Here, $X=1.5 \mathrm{~km} / \mathrm{s}, \eta=0.1$, and $V_{\text {nmo }}(0)=2.0$ $\mathrm{km} / \mathrm{s}$. Again, the responses for the VTI medium (black) are superimposed on the corresponding isotropic constant-velocity operators (gray). 
Distance $(\mathrm{km})$
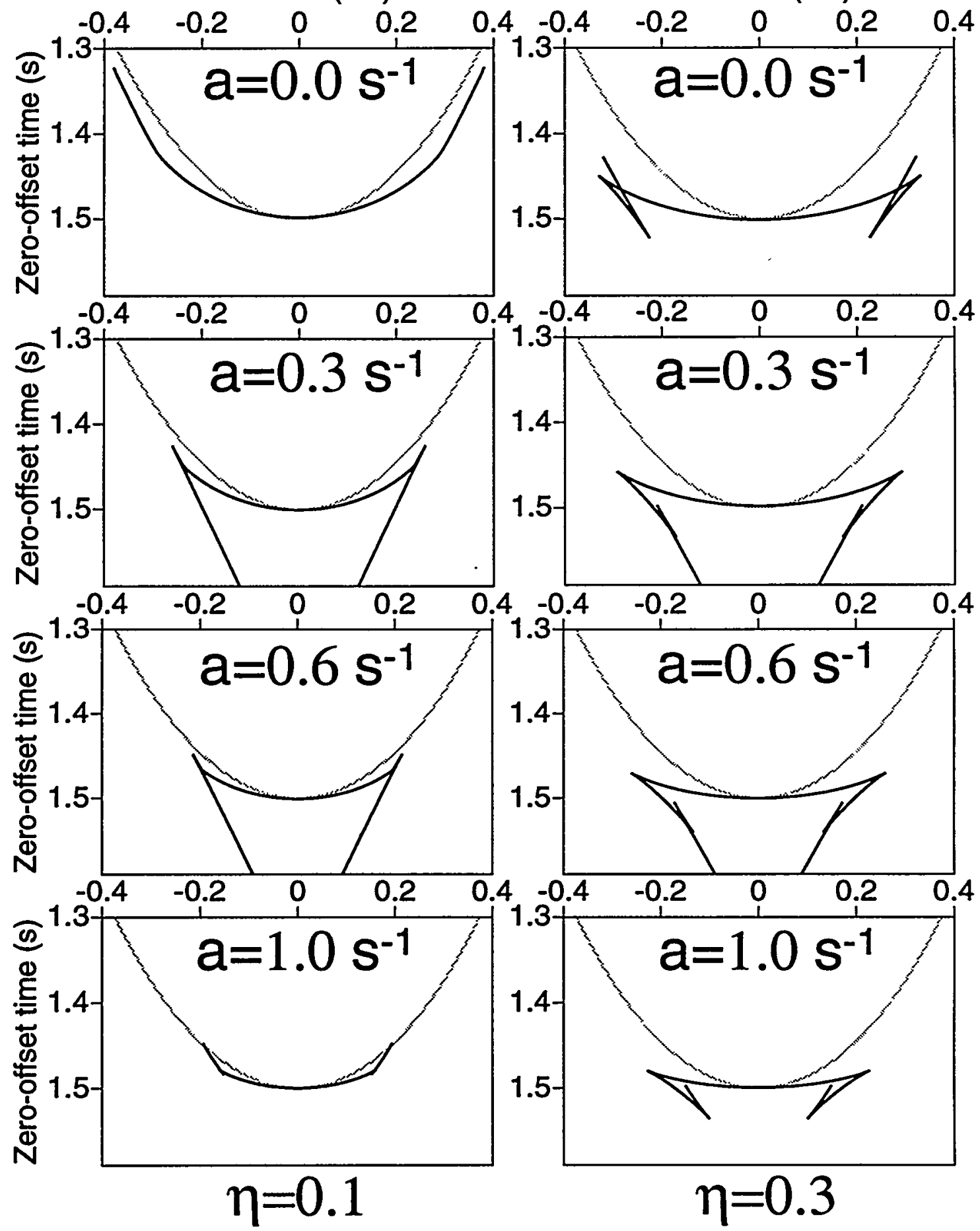

FIG. 7. DMO impulse responses in vertically inhomogeneous VTI media for various vertical velocity gradients. Here, offset $=1.5 \mathrm{~km}, t_{n}=1.5 \mathrm{~s}$, and $V_{\text {nmo }}(0)$ at the surface is $1.5 \mathrm{~km} / \mathrm{s}$. $\eta$ for the left column of plots equals 0.1 and for the right column equals 0.3 (black curves). The corresponding isotropic constant-velocity operators are shown in gray. 

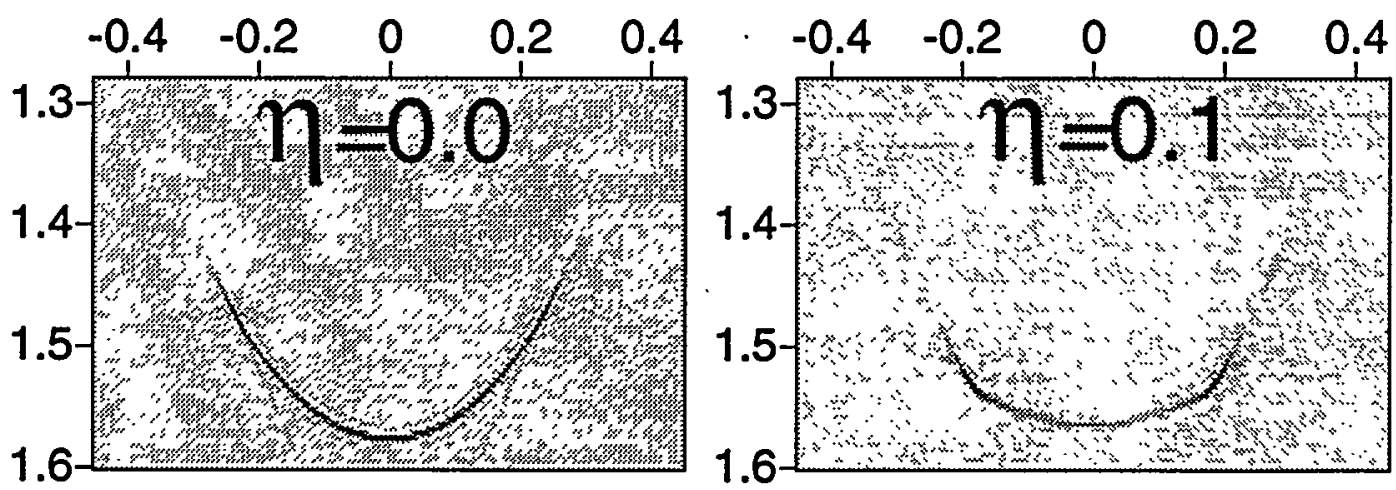

FIG. 8. DMO impulse responses in inhomogeneous isotropic $(\eta=0.0)$ and VTI media $(\eta=0.1)$. Here, offset $=1.5 \mathrm{~km}, t_{n}=1.5 \mathrm{~s}$, and $V_{\text {nmo }}(0)=1.5+0.6 z \mathrm{~km} / \mathrm{s}$.

ellipses, similar approximations will not work effectively in VTI media, especially for dips exceeding 30 degrees.

\section{PRESTACK MIGRATION IMPULSE RESPONSES}

Applying a zero-offset time migration to a DMO impulse response simulates a prestack time-migration impulse response for the same offset as that used in generating the DMO impulse response. This holds for VTI media, as well as isotropic media.

Figure 9 shows such prestack migration impulse responses for an offset of $1.5 \mathrm{~km}$ (the same offset used in generating the responses in Figure 3). The time migration used here is the Gazdag (1978) phase-shift method modified for VTI media. Despite the complicated form of the DMO impulse responses in VTI media (e.g., the triplication for larger $\eta$ ), the time-migration impulse responses are smooth. This can be expected because migration impulse responses represent wavefronts, and for $P$ waves in VTI media the wavefronts are known to be smooth (i.e., they do not include triplications). Further, the width of the migration responses depends on $\eta$ : from equation (2), negative $\eta$ implies a smaller horizontal velocity than $V_{\text {nmo }}(0)$, resulting in a squeezed migration response, whereas positive $\eta$ implies a larger horizontal velocity, resulting in a stretched version. For $\eta$ equal zero (isotropic or elliptically anisotropic media), the response shape is simply the familiar ellipse.

\section{APPLICATION TO A REFLECTOR MODEL}

Although this DMO algorithm handles $v(z)$ VTI media, the example here pertains to homogeneous media. The reason for this simplification is to show the significance of anisotropy and its influence on the data without any influence of inhomogeneity. 


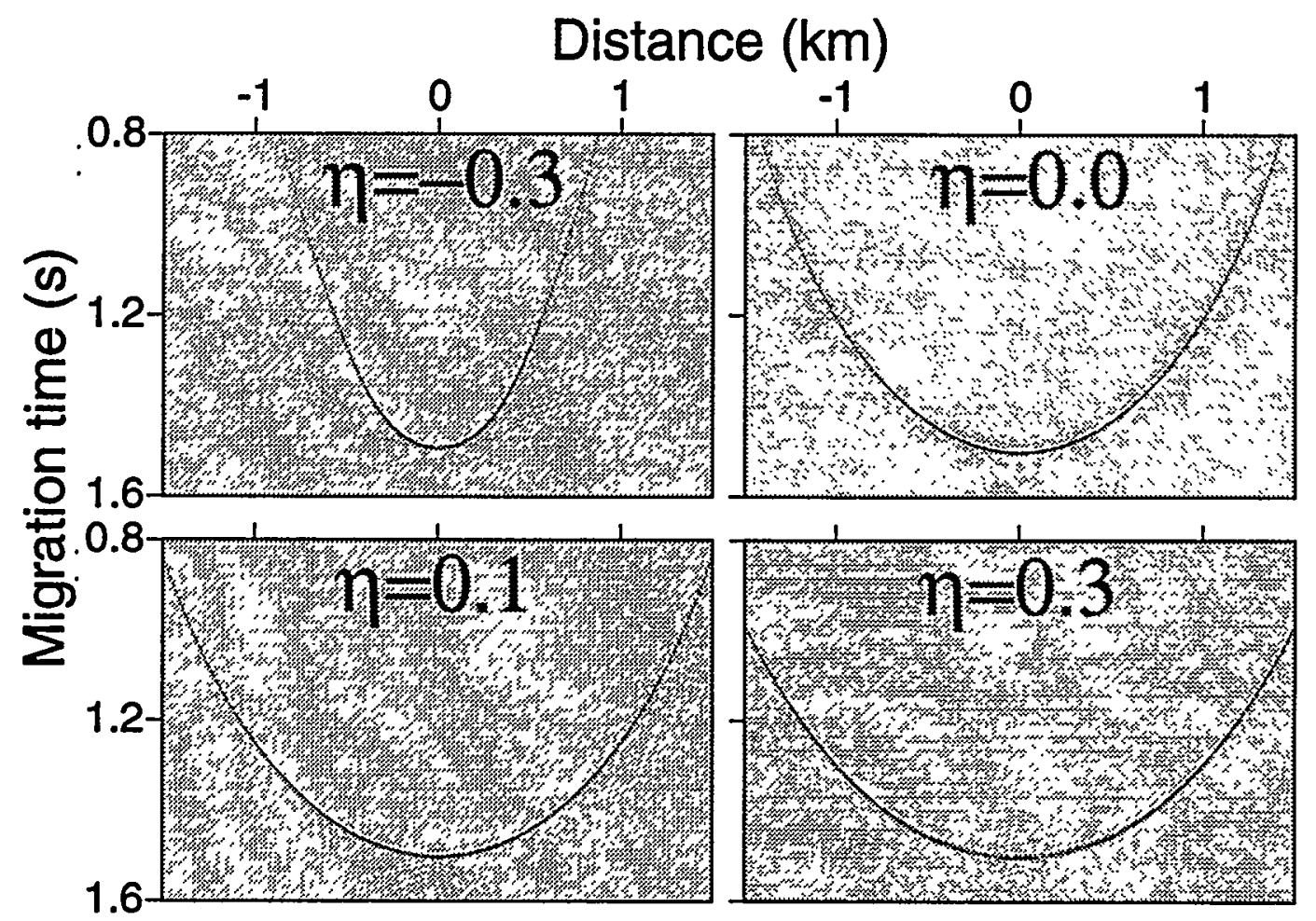

FIG. 9. Prestack migration impulse responses generated from the DMO impulse responses in Figure 2 for several $\eta$ values. Here again, offset $=1.5 \mathrm{~km}, t_{n}=1.5 \mathrm{~s}$, and $V_{\mathrm{nmo}}(0)=2.0 \mathrm{~km} / \mathrm{s}$. 


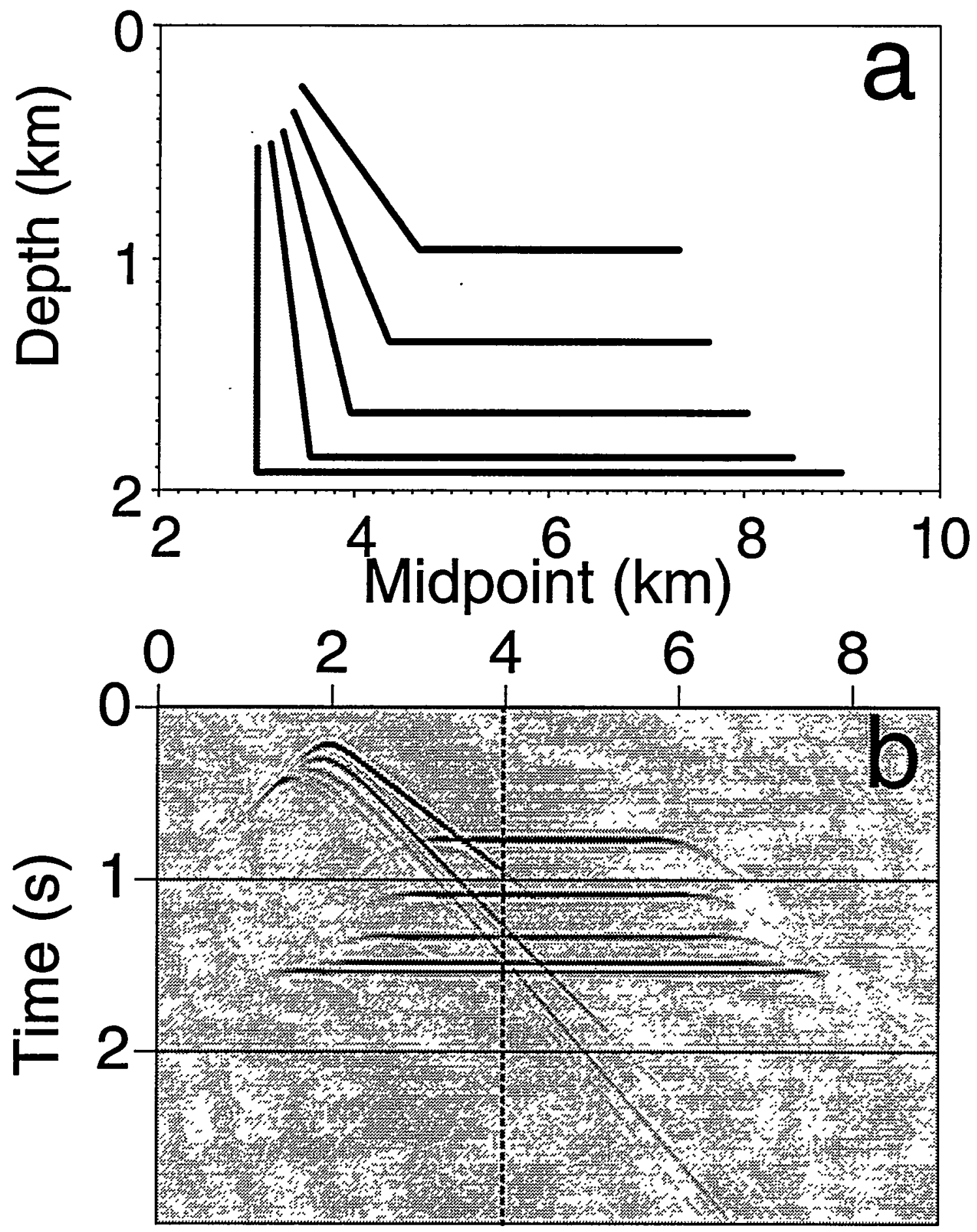

Frg. 10. (a) Reflector model containing reflectors dipping at $0,30,45,60,75$, and 90 degrees. (b) Zero-offset seismogram generated from the model. The medium is homogeneous VTI with $V_{P 0}=2.5 \mathrm{~km} / \mathrm{s}, \epsilon=0.3$, and $\delta=0.1(\eta=0.167)$. The dashed line shows the location of the CMP gather in Figure 11. 
Figure $10 \mathrm{~b}$ shows the zero-offset synthetic section for the homogeneous model in Figure 10a. The section has 401 traces, corresponding to $401 \mathrm{CMP}$ locations, at a spacing of $0.025 \mathrm{~km}$. The medium above the reflectors is VTI with $V_{P 0}=2.5 \mathrm{~km} / \mathrm{s}$, $\epsilon=0.3$, and $\delta=0.1$. For NMO and DMO purposes, such media can also be expressed by $\eta=0.167$ and $V_{\text {nmo }}(0)=2.78 \mathrm{~km} / \mathrm{s}$. Figure 11a shows a 31-fold CMP gather prior to TZO for the CMP location marked by the dashed vertical line in Figure 10b. Reflections from dipping interfaces can be identified by their higher velocity (i.e., smaller moveout).

First, let us examine application of the conventional processing sequence designed for isotropic media. Figure 11b shows the same CMP gather of Figure 11a after an isotropic TZO (NMO and DMO) using $V_{\text {nmo }}(0)$, which approximates the stacking velocity obtained from velocity analysis for offset-to-depth ratio $X / D<1$. Although the reflections from horizontal interfaces are flattened at the near offsets, the nonhyperbolic moveout behavior caused by anisotropy leads to deviations at the far offsets. Such errors cannot be corrected using a hyperbolic moveout correction, and therefore a mute procedure would typically be applied to the far offsets to properly stack the data (Anderson et al., 1995). On the other hand, reflections from dipping reflectors have even larger errors, which are caused by ignoring anisotropy in DMO for the VTI medium. Such errors are described in detail by Larner (1993) and Tsvankin (1995).

Using anisotropic ray-tracing, DMO with the correct medium parameters flattens the moveout of all events properly at all offsets, as demonstrated in Figure 11c. Both the nonhyperbolic moveout and the dip moveout have been properly corrected. Futhermore, Figure 11d shows the same perfect TZO correction using the correct values of $V_{\text {nmo }}(0)$ and $\eta$, but with erroneous $V_{p 0}=V_{\text {nmo }}(0)$ [from equations (2) and (1)], corresponding to erroneous values $\delta=0$ and $\epsilon=0.167$. This confirms results obtained by Alkhalifah and Tsvankin (1995) that NMO and DMO (or TZO), as well as time migration, depend only on $\eta$ and $V_{\text {nmo }}(0)$.

\section{FIELD-DATA EXAMPLE}

Figure 12 shows a seismic line from offshore Africa provided by Chevron Overseas Petroleum, Inc. The line was processed using a sequence of conventional NMO, DMO, and time-migration algorithms without taking anisotropy into account. While horizontal and mildly dipping reflectors are imaged well, steep fault-plane reflections (such as the one at a time of 1.5-2 s to the left of CMP 1000) show weak amplitude. Velocity analysis at CMP locations below the gray bar in Figure 12 shows a pronounced vertical velocity gradient of about $0.7 \mathrm{~s}^{-1}$.

As Alkhalifah and Tsvankin (1995) showed, and as we saw in Figure 1, these data are influenced by the presence of anisotropy. The influence of the anisotropy on the DMO operation is sufficiently large that it clearly shows (in Figure 1) even in the presence of vertical inhomogeneity, which usually has an opposite action on DMO (Gonzalez et al., 1992; Larner, 1993). Based on an inversion procedure for a homogeneous VTI medium, Alkhalifah and Tsvankin (1995) obtain an estimate of 


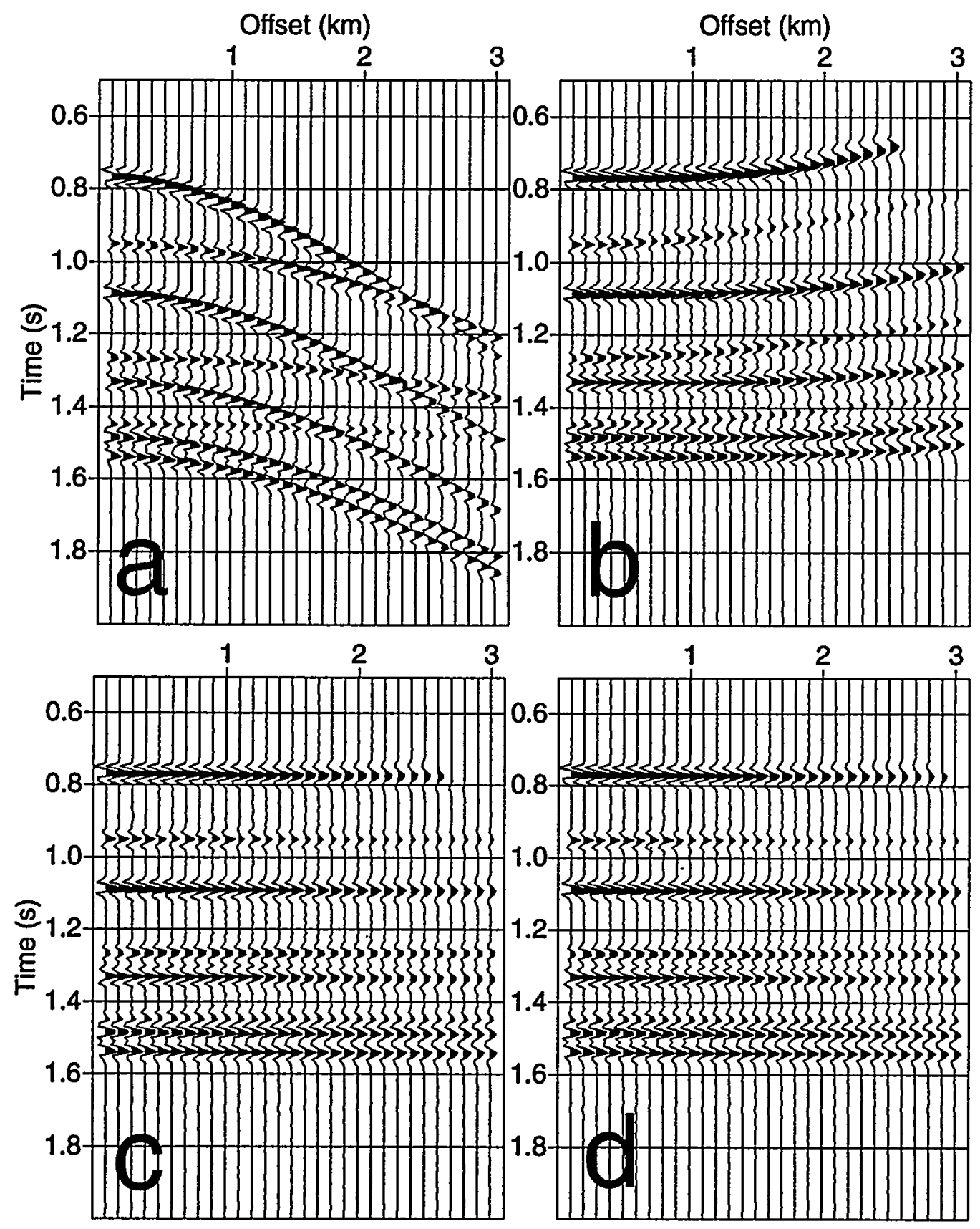

FIG. 11. (a) Uncorrected CMP gather for the model shown in Figure 10a. (b) Gather after isotropic TZO using $V_{\text {nmo }}(0)$. (c) Gather after anisotropic TZO with the actual anisotropy parameters. (d) Gather after anisotropic TZO with the correct values of $\eta$ and $V_{\text {nmo }}(0)$, but with incorrect values of $V_{p 0}\left(V_{p 0}=V_{\text {nmo }}(0)=2.65 \mathrm{~km} / \mathrm{s}\right), \delta(\delta=0)$, and $\epsilon(\epsilon=0.167)$. The three dipping reflectors shown here are at $0.96 \mathrm{~s}$ (30-degree dip), at $1.26 \mathrm{~s}$ (45-degree dip), and at $1.44 \mathrm{~s}$ (60-degree dip). 


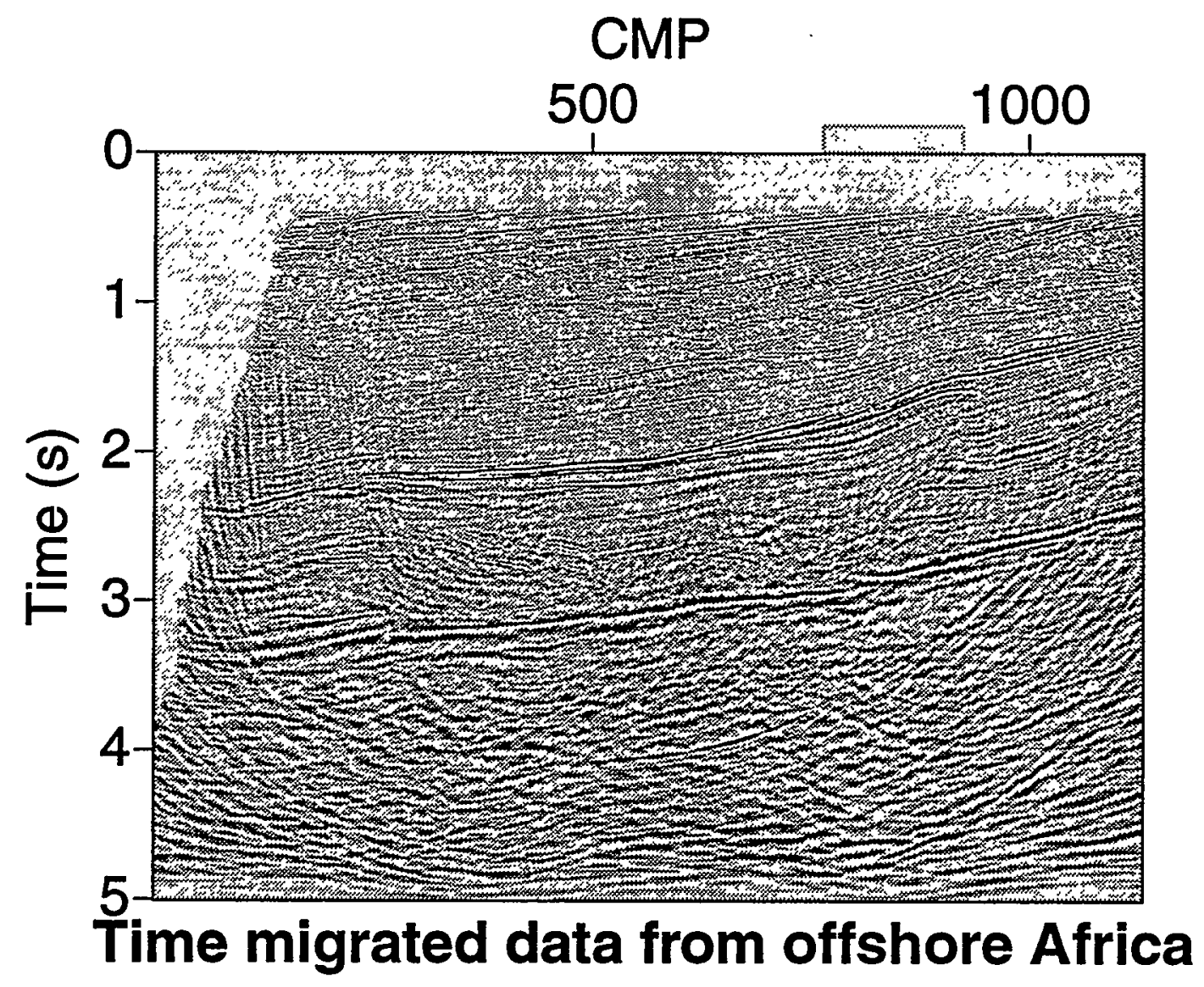

FIG. 12. Time-migrated seismic line (offshore Africa). The gray bar to the left of CMP 1000 shows the CMP locations that contain the unmigrated data from the fault of interest. 
$\eta=0.07$ for the area under the gray bar of Figure 12. Since an increase in vertical velocity with depth tends to reduce the estimated value of $\eta$, I expect that this estimate is lower than the true value attributable solely to the anisotropy of the medium. Therefore, I shall call the value computed using the homogeneous model the "effective $\eta$." Application of anisotropic DMO based on the effective $\eta=0.07$ allowed Alkhalifah and Tsvankin (1995) to improve the results of the conventional isotropic DMO. However, as they explained, their effective $\eta$, while useful for DMO, may be inappropriate for migration.

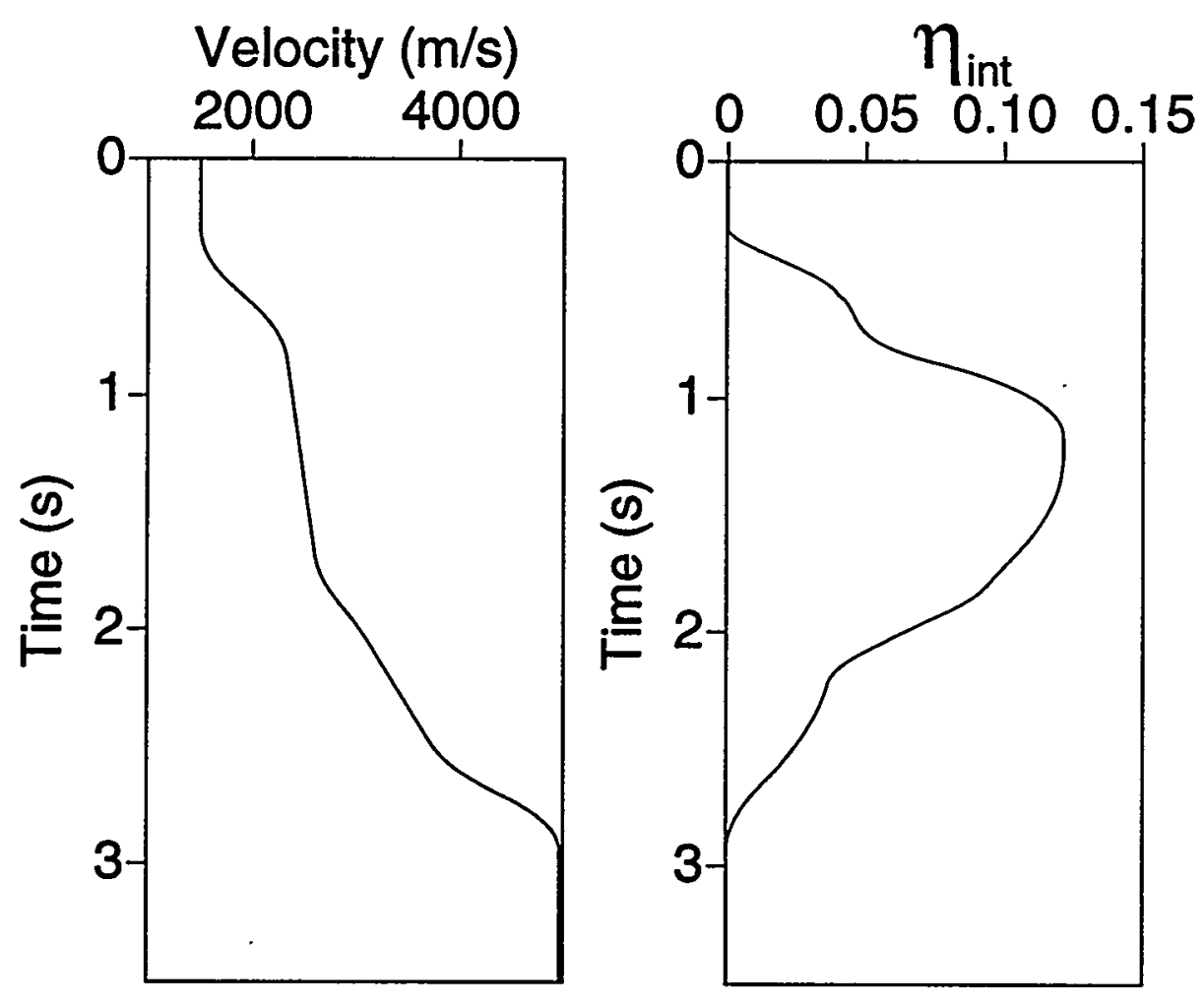

FIG. 13. Interval values $v_{\text {int }}$ and $\eta_{\text {int }}$ as a function of vertical time.

To take full advantage of the DMO approach developed here, I did a $v(z)$ inversion, based on equation (19) of Alkhalifah and Tsvankin (1995), in order to obtain interval values $v_{\text {int }}(\tau)$ and $\eta_{\text {int }}(\tau)$, where $\tau$ is vertical time. Such interval values of the anisotropy parameter can then be used in the migration step.

Figure 13 shows $v_{\text {int }}(\tau)$ and $\eta_{\text {int }}(\tau)$, obtained from the $v(z)$ VTI inversion. This inversion is accomplished by estimating the NMO velocity as a function of ray parameter for the dipping fault at five different points along the fault, corresponding to five different vertical times. I assume no lateral velocity variation in the region of the fault; however, mild lateral velocity variation should not be a problem for the 
inversion: most DMO algorithms, while based on lateral homogeneity, still produce practical results where lateral velocity variation is smooth. The continuous representation shown in Figure 13 is generated by linear interpolation of the velocities for both the mildly dipping reflectors (for simplicity I refer to them as horizontal reflectors) and the fault. In the water layer, $v_{\text {int }}$ is equal to $1.5 \mathrm{~km} / \mathrm{s}$ and $\eta_{\text {int }}$ is equal to zero. The accuracy of these estimated curves of $v_{\text {int }}$ and $\eta_{\text {int }}$ depends on the accuracy of the stacking velocity estimates for both dipping and horizontal reflectors (Alkhalifah and Tsvankin, 1995). The idea underlying the inversion is that the $v_{\text {int }}(\tau)$ and $\eta_{\text {int }}(\tau)$ functions obtained are those that best focus the dipping fault and the horizontal reflectors at the same stacking (or NMO) velocity, for each vertical time at which velocity measurements are made.

In Figure 13, where $\eta_{\text {int }}=0$, the medium is either isotropic or elliptical anisotropic. Because elliptical anisotropy for P-waves is not typical in the subsurface (Thomsen, 1986), one might infer that, where $\eta_{\text {int }}=0$, the medium is more likely to be isotropic. The region above $2 \mathrm{~s}$, which exhibits positive values of $\eta_{\text {int }}$, corresponds to a shale formation, which is usually transversely isotropic and may thus be the major source of anisotropy in the data.

Figure 14 shows the result of VTI DMO applied to the data, based on the derived functions $v_{\text {int }}(\tau)$ and $\eta_{\text {int }}(\tau)$. We can clearly see the presence of a fault, which is dipping at an average angle of about 45 degrees. This angle exceeds the dips included in the first branch of the DMO impulse response. Recall that this first branch is the only branch that can be approximated by a stretched ellipse. Therefore, we cannot expect that for this medium isotropic constant-velocity or $v(z)$ DMO or a stretched version of the DMO ellipse would perform as well as has the $v(z)$ anisotropic DMO, here.

This expectation is supported by Figure $15 \mathrm{a}$ and $15 \mathrm{~b}$. Figure $15 \mathrm{a}$ shows the zerooffset section obtained by applying isotropic homogeneous DMO, and Figure 15b shows that obtained by applying isotropic $v(z)$ DMO. The isotropic homogeneous DMO used here is the frequency-wavenumber method of Hale (1984), and the $v(z)$ isotropic DMO is Artley and Hale's (1994) isotropic version of the DMO method described in this paper. Clearly, the fault is poorly imaged by both isotropic algorithms, while VTI DMO yields a much better result (Figure 14). Figure 16 shows a representative VTI DMO operator used for these data. The shape is far from the isotropic ellipse or a squeezed version of it.

Not only did the $v(z)$ VTI DMO do a better job of imaging the dipping fault, it also improved the continuity of horizontal reflectors (e.g., the regions pointed to by the arrows in Figures 14 and 15) by correcting for nonhyperbolic moveout. Figure 17 shows CMP gathers at CMP location 700 after (a) $v(z)$ VTI DMO, (b) homogeneous isotropic DMO, and (c) $v(z)$ isotropic DMO. The same NMO correction was used for all three DMO examples. It is based on the stacking velocities obtained from a typical velocity analysis. All reflections shown in this figure are from horizontal reflectors. Of the three approaches, only the $v(z)$ VTI DMO aligns the reflection just above $2 \mathrm{~s}$ 


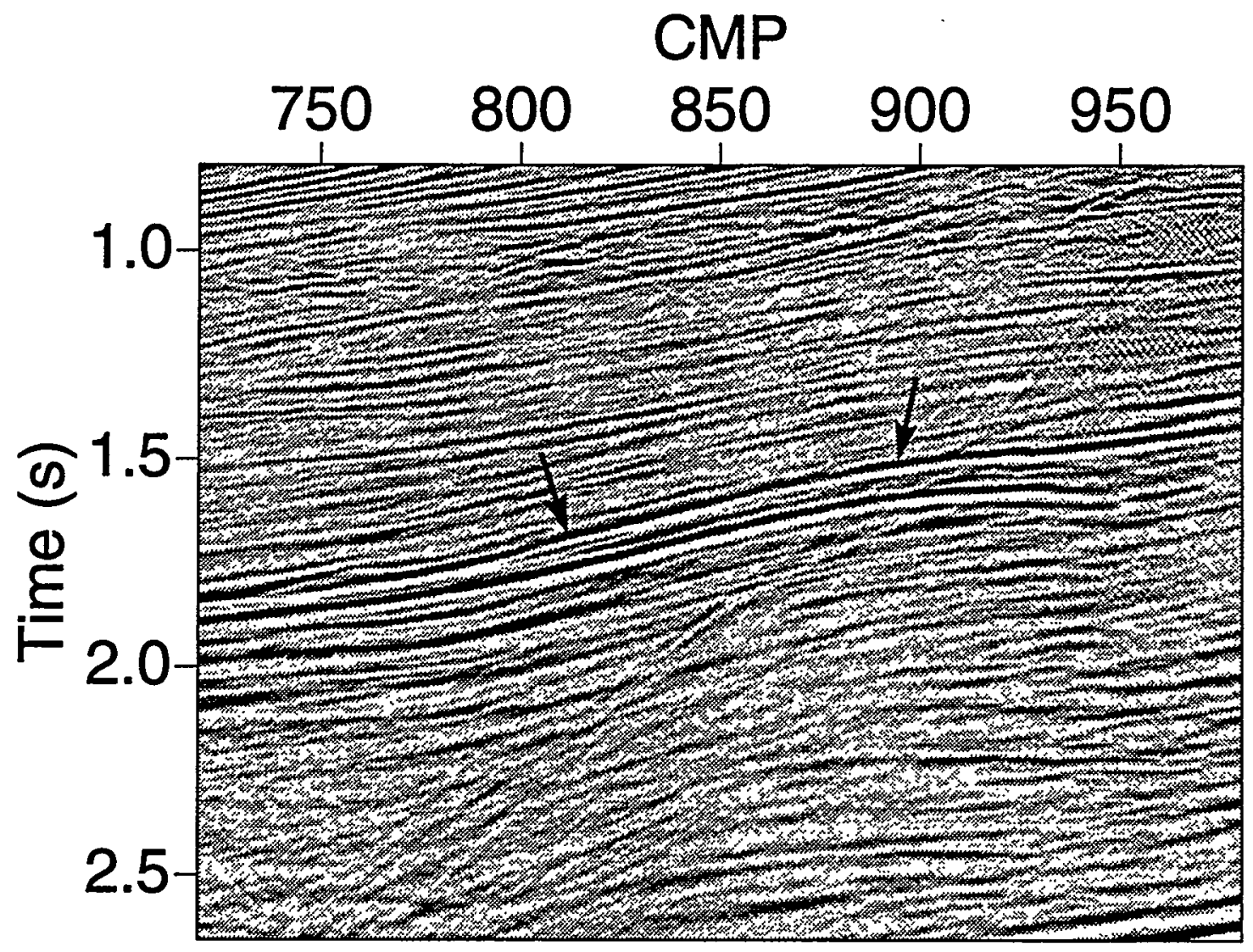

FIG. 14. Zero-offset section after a $v(z)$ anisotropic DMO using the parameters in Figure 13. The NMO correction is based on the velocities obtained from the conventional velocity analysis. The arrows point to regions of improved reflection continuity relative to that in Figure 15. 


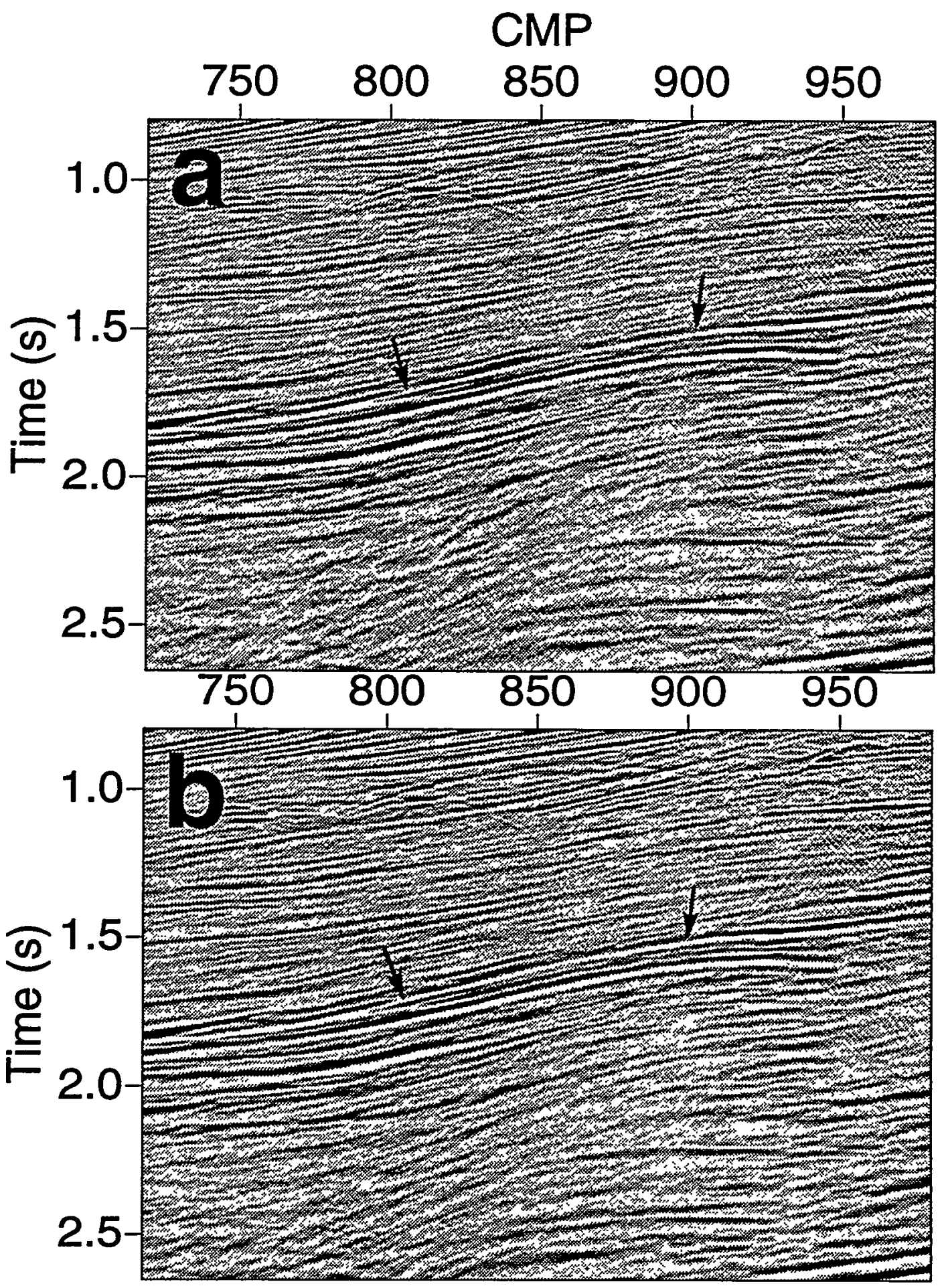

Frg. 15. Zero-offset section after (a) homogeneous isotropic DMO, and (b) $v(z)$ isotropic DMO. The NMO correction is the same as in Figure 14. 


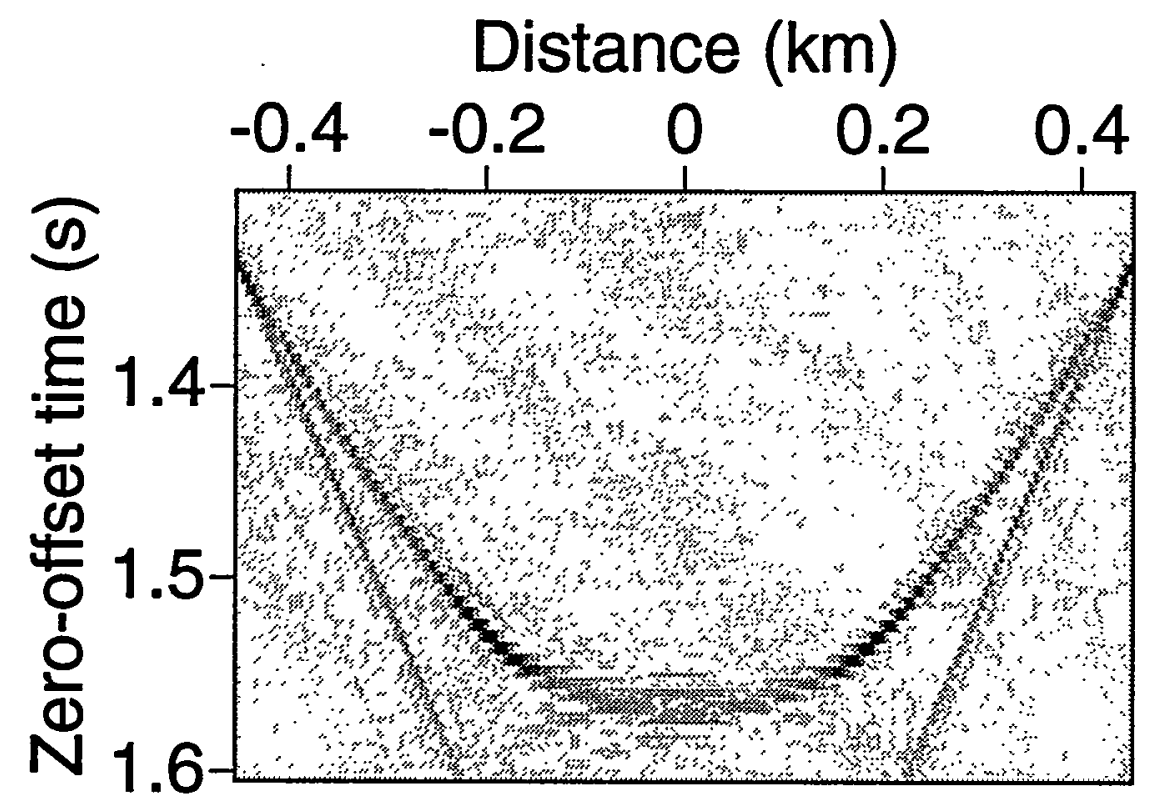

FIG. 16. VTI DMO impulse response for the parameters in Figure 13. The offset is $1.5 \mathrm{~km}$. 
for all offsets.

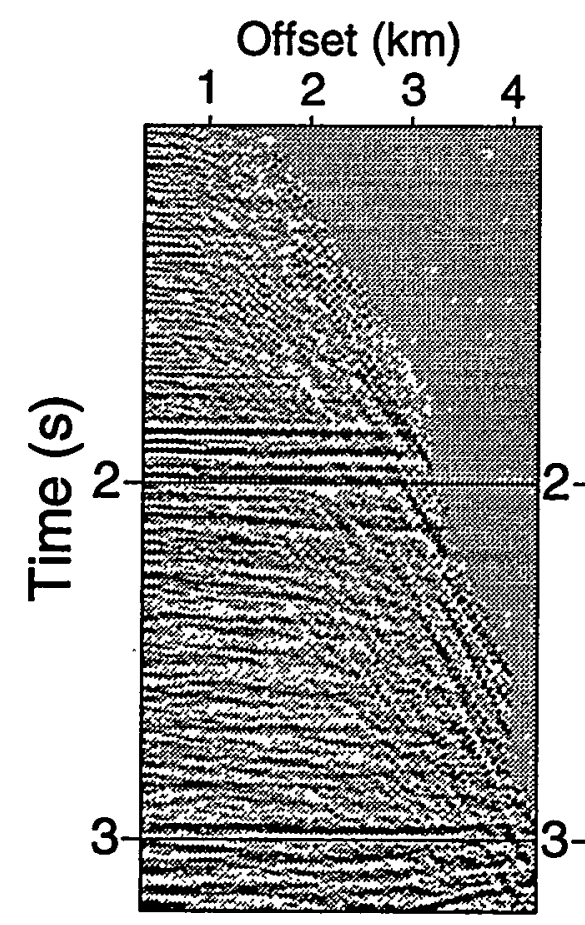

(a)

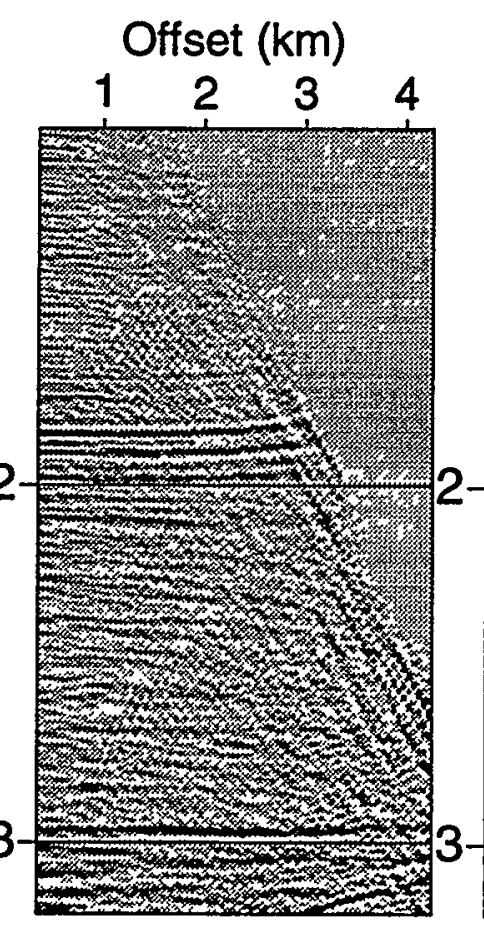

(b)

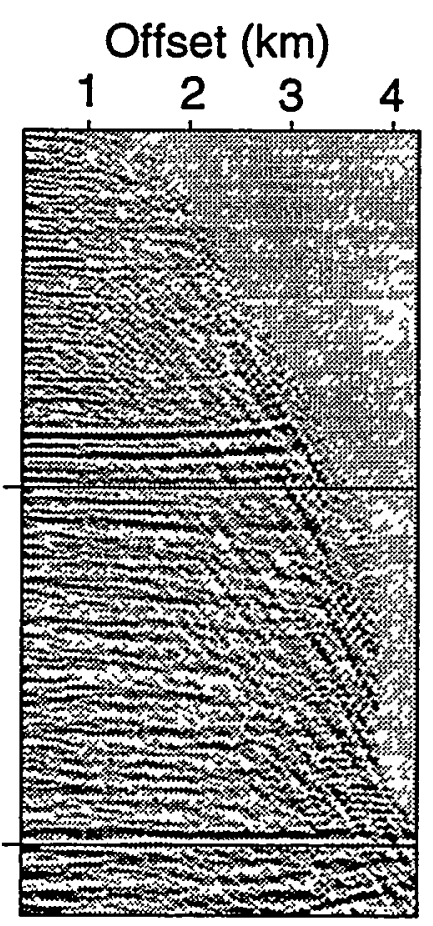

(c)

FIG. 17. CMP gathers for CMP location 700 after (a) $v(z)$ anisotropic DMO, (b) homogeneous isotropic DMO, and (c) $v(z)$ isotropic DMO. The NMO correction, based on the velocities obtained from velocity analysis, is the same for all three.

CMP location 700, in Figure 17, is to the left of the region of the imaged fault (see Figure 14). Figure 18 shows CMP location 845 , which contains the imaged faultplane reflection after (a) $v(z)$ VTI DMO, (b) homogeneous isotropic DMO, and (c) $v(z)$ isotropic DMO. The arrows point to the time of the fault reflection. Whereas the result from $v(z)$ VTI DMO aligns the weak fault clearly, the dipping event after isotropic DMO is misaligned.

Figure 19a shows a close-up of the fault imaged with phase-shift anisotropic time migration (using the inverted parameters of Figure 13) applied to the stack obtained from the $v(z)$ VTI DMO algorithm. For comparison, Figure 19b shows the result of conventional processing: phase-shift isotropic time migration applied to the zero-offset section obtained from the isotropic homogeneous DMO. Figure 19 gives a clear picture of the benefit of taking anisotropy into account in DMO prior to doing migration (the influence of anisotropy on the migration step is not large, here). 


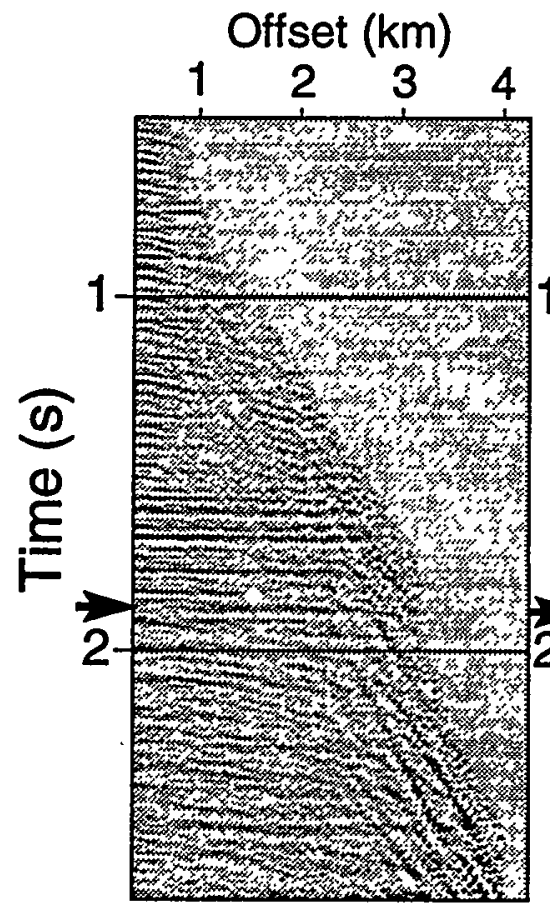

(a)

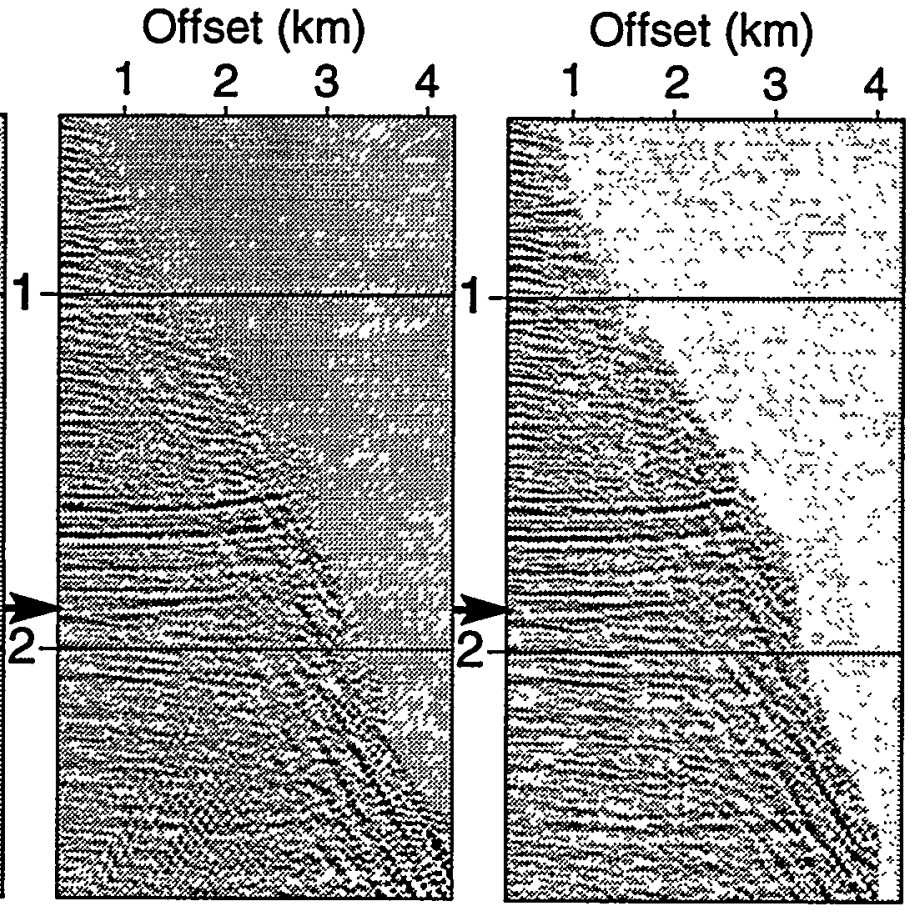

(b)

(c)

FIG. 18. CMP gathers for CMP location 845 after (a) $v(z)$ anisotropic DMO, (b) homogeneous isotropic DMO, and (c) $v(z)$ isotropic DMO. The NMO correction, based on the velocities obtained from velocity analysis, is the same for all three sections. The arrow points to the time location of the dipping fault reflection. 


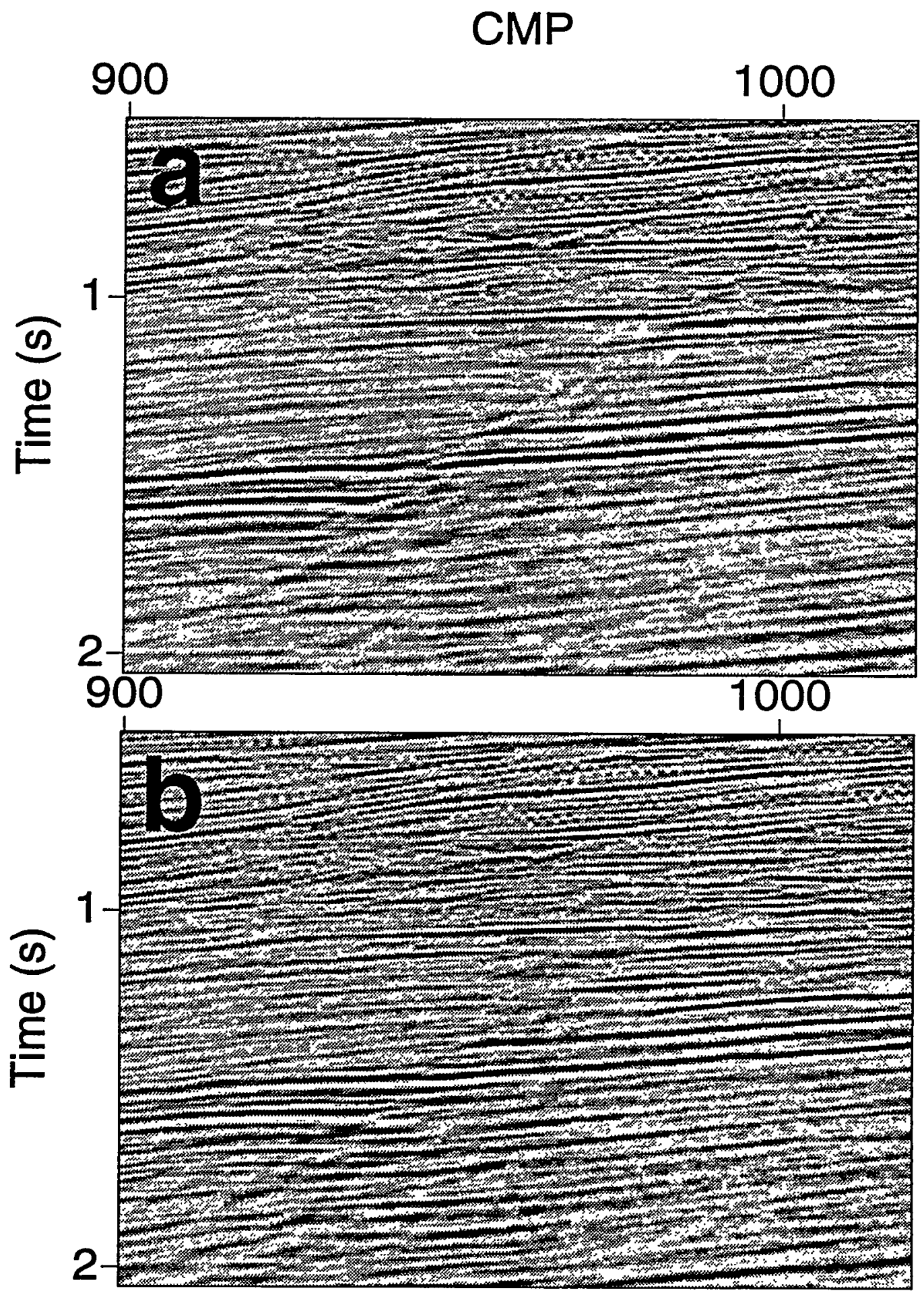

FIG. 19. Time migrated section using (a) anisotropic phase-shift migration of the data shown in Figure 14 using the parameters shown in Figure 13, and (b) isotropic phase-shift migration of the data shown in Figure 15a. 


\section{CONCLUSIONS}

Accurate transformation of prestack data to zero-offset, where the subsurface is transversely isotropic, must fully honor the anisotropy. Specifically, TZO based on anisotropic ray tracing can accurately treat data from TI media, with no dip or offset limitations. While the usual NMO and DMO sequence based on hyperbolic moveout correction ignores the sizable nonhyperbolic moveout associated with horizontal events in VTI media, the scheme presented here provides a method for exactly computing traveltimes and using that information to apply accurate TZO for arbitrary depth-variable anisotropy functions.

Similar to isotropic DMO operators, the impulse response in VTI media is practically velocity-independent; the shape of the response depends mainly on $\eta$. For $\eta=0$ (isotropic or elliptically anisotropic media), the impulse response is an ellipse, but as $\eta$ varies, the response takes on shapes that can differ substantially from that for elliptical media. For example, impulse responses in homogeneous VTI media with typical values of anisotropy $(\eta>0)$ can include triplications that arise for reflector dip of about 30 degrees. The presence of the triplications exemplifies the large differences between DMO impulse responses for VTI media and those for isotropic media. Moreover, the complex shape of the impulse response in VTI media leads to an amplitude distribution along the DMO operator that differs significantly from that in isotropic media. With such large differences, we can expect isotropic DMO to be inadequate for typical VTI media. For example, stretching of the isotropic DMO impulse response clearly will fail to emulate the VTI DMO response for dips beyond about 30 degrees, where the triplications begin.

The impulse responses in VTI media typically deviate more from the isotropic operator than do those attributable solely to vertical inhomogeneity. For example, $v(z)$ isotropic DMO impulse responses can be well approximated by squeezed ellipses up to and beyond 90 degrees reflector dips (Hale and Artley, 1994). Thus, the influence of typical anisotropy (i.e., $\eta \simeq 0.1$ ) on DMO exceeds that of typical vertical inhomogeneity (i.e., average velocity gradient $a=0.6$ ).

As we saw in the field example, isotropic DMO cannot properly focus dipping reflectors where the inversion results indicate that the medium is anisotropic. On the other hand, $v(z)$ VTI DMO does focus such reflectors, and, because it also takes nonhyperbolic moveout into account, it can even improve the focussing of horizontal reflections.

It remains to be seen to what degree of confidence derived values of $\eta(z)$ can be used to augment lithologic interpretation. In any case, by introducing the new anisotropy parameter $\eta$, we have seen considerably improved DMO operation in the presence of VTI media. It could be argued that the improvements in imaging are simply the result of the introduction of an extra parameter (fudge factor), that offers an added degree of freedom in the imaging. However, the theoretical development by Alkhalifah and Tsvankin (1995) provides a sound basis for viewing this parameter 
as appropriate for processing of data from VTI media and potentially of value in lithologic interpretation.

\section{ACKNOWLEDGMENTS}

I am grateful to Ken Larner, Ilya Tsvankin, John Anderson, and Craig Artley for helpful discussions. I am also grateful to Ken Larner for his critical review of this paper. I thank Tagir Galikeev for preprocessing the data, and John Toldi and Chris Dale of Chevron Overseas Petroleum, Inc. for providing the field data. Thanks are due to the Center for Wave Phenomena, Colorado School of Mines, for its technical support, and also to KACST, Saudi Arabia, for its financial support. Financial support for this work also was provided in part by the United States Department of Energy, grant number DE-FG02-89ER14079 (this support does not constitute an endorsement by DOE of the views expressed in this paper).

\section{REFERENCES}

Alkhalifah, T., 1995, Velocity analysis using nonhyperbolic moveout in transversely isotropic media: Center for Wave Phenomena, Colorado School of Mines (CWP167).

Alkhalifah, T., and Larner, K., 1994, Migration errors in transversely isotropic media: Geophysics, 59, 1405-1418.

Alkhalifah, T., and Tsvankin, I., 1995, Velocity analysis for transversely isotropic media: Geophysics, in press.

Anderson, J., and Tsvankin, I., 1995, Dip-moveout processing by Fourier transform in anisotropic media: Geophysics, submitted.

Anderson, J., Alkhalifah, T., and Tsvankin, I., 1995, Fowler DMO and Time Migration for Transversely Isotropic Media: Geophysics, submitted.

Artley, C. T., and Hale, D., 1994, Dip moveout processing for depth-variable velocity: Geophysics, 59, 610-622.

Banik, N. C., 1984, Velocity anisotropy of shales and depth estimation in the North Sea basin: Geophysics, 49, 1411-1419.

Červený, V., 1972, Seismic rays and ray intensities in inhomogeneous anisotropic media: Geophys. J. R. Astr. Soc., 29, 1-13.

Forel, D., and Gardner, G.H.F., 1988, A three-dimensional perspective on twodimensional dip moveout: Geophysics, 53, 604-610.

Gazdag, J., 1978, Wave equation migration with the phase-shift method: Geophysics, 43, 1342-1351.

Gonzalez, A., Levin., F. K., Chambers, R. E., and Mobley, E., 1992, Method of correcting 3-D DMO for the effects of wave propagation in an inhomogeneous earth, 62nd Ann. Internat. Mtg., Soc. Expl. Geophys., Expanded Abstracts, 966969. 
Hake, H., Helbig, K., and Mesdag, C. S., 1984, Three-term Taylor series for $t_{2}-x^{2}$ curves over layered transversely isotropic ground: Geophys. Prosp., 32, 828-850.

Hale, D., 1984, Dip-moveout by Fourier transform: Geophysics, 49, 741-757.

Hale, D., and Artley, C. T., 1993, Squeezing DMO for depth-variable velocity: Geophysics, 58, 257-264.

Jakubowicz, H., 1990, A simple efficient method of dip-moveout correction: Geophys. Prosp., 38, 221-245.

Larner, K., 1993, Dip-moveout error in transversely isotropic media with linear velocity variation in depth: Geophysics, 58, 1442-1453.

Thomsen, L., 1986, Weak elastic anisotropy: Geophysics, 51, 1954-1966.

Tsvankin, I., 1995, Normal moveout from dipping reflectors in anisotropic media: Geophysics, 60, 268-284.

Tsvankin, I., and Thomsen, L., 1994, Nonhyperbolic reflection moveout in anisotropic media: Geophysics, 59, 1290-1304.

Uren, N.F., Gardner, G.H.F., and McDonald, J.A., 1990, Dip moveout in anisotropic media: Geophysics, 55, 863-867.

\section{APPENDIX A: ANISOTROPIC RAY TRACING FOR TZO}

Červený (1972) has derived an efficient ray-tracing system for general inhomogeneous, anisotropic media. This system is based on solving for the partial derivatives of the eigenvalues of the Christoffel $3 \times 3$ matrix, $\Gamma$, rather than solving for the eigenvalues themselves. The components of this matrix are given by

$$
\Gamma_{i k}\left(x_{s}, p_{i}\right)=a_{i j k l}\left(x_{s}\right) p_{j} p_{l},
$$

with

$$
\begin{aligned}
p_{i} & =\frac{\partial \tau}{\partial x_{i}}, \\
a_{i j k l} & =c_{i j k l} / \rho,
\end{aligned}
$$

where $p_{i}$ are the three components of the slowness vector, $\tau$ is the traveltime along the ray, $\rho$ is bulk density, $x_{s}$ are the cartesian coordinates for position along the ray, $\mathrm{s}=1,2,3$, and $c_{i j k l}$ are elastic coefficients, in general, functions of $x_{s}$. In the above expression for $\Gamma_{i k}$, the summation convention for repeated indices is used.

From Červený (1972), the ray-tracing system for general inhomogeneous anisotropic media is given by

$$
\begin{gathered}
\frac{d x_{i}}{d t}=a_{i j k l} p_{l} g_{j} g_{k}, \\
\frac{d p_{i}}{d t}=-\frac{1}{2} \frac{\partial a_{n j k l}}{\partial x_{i}} p_{n} p_{l} g_{j} g_{k}
\end{gathered}
$$


where $g_{i}$ are the components of the eigenvector $g$ (the direction of particle motion).

In 2-D, all out-the-plane components vanish, namely all components with subscript 2. The eigenvectors are then calculated as follows.

$$
\begin{aligned}
& g_{1} g_{1}=\frac{\Gamma_{33}-G}{\Gamma_{11}+\Gamma_{33}-2 G}, \\
& g_{3} g_{3}=\frac{\Gamma_{11}-G}{\Gamma_{11}+\Gamma_{33}-2 G},
\end{aligned}
$$

and

$$
g_{1} g_{3}=\frac{-\Gamma_{13}}{\Gamma_{11}+\Gamma_{33}-2 G}
$$

where $G$ is the eikonal (described below) and $G=1$ along the ray.

For TZO purposes the vertical axis, $x_{3}$, is conveniently replaced with vertical time, $\tau$. This can be accomplished by substituting the following equation

$$
\frac{d \tau}{d x_{3}}=\frac{1}{V_{p 0}}=\frac{1}{\sqrt{a_{3333}}}
$$

into the ray-tracing system. Further, since we are considering a $v(z)$ medium, the ray parameter, $p_{1}=p$, is constant and the ray tracing system in 2-D reduces to

$$
\begin{aligned}
& \frac{d x_{1}}{d t}=a_{1 j k l} p_{l} g_{j} g_{k}, \\
& \frac{d \tau}{d t}=\frac{a_{3 j k l}}{V_{p 0}} p_{l} g_{j} g_{k},
\end{aligned}
$$

and $p_{3}$ is obtained using (Larner, 1993)

$$
\begin{aligned}
1=0.5\left\{\left(a_{1111}+a_{1313}\right) p^{2}+\left(a_{3333}+a_{1313}\right) p_{3}^{2}+\left\{\left[\left(a_{1111}-a_{1313}\right) p^{2}-\left(a_{3333}-a_{1313}\right) p_{3}^{2}\right]^{2}\right.\right. \\
\left.\left.+4\left(a_{1133}+a_{1313}\right)^{2} p^{2} p_{3}^{2}\right\}^{\frac{1}{2}}\right\} .
\end{aligned}
$$
by

Also, both the phase velocity and phase angle need to calculated. They are given

$$
V(p)=\frac{1}{\sqrt{p^{2}+p_{3}^{2}}}
$$

and

$$
\theta=\sin ^{-1}(p V),
$$

respectively. Both values are tabulated and used in solving the system of five equations for the ray trio mentioned prior to equation (3). 